\title{
Luminescent solar concentrators. 2: Experimental and theoretical analysis of their possible efficiencies
}

\author{
J. S. Batchelder, A. H. Zewail, and T. Cole
}

\begin{abstract}
Experimental techniques are developed to determine the applicability of a particular luminescing center for use in a luminescent solar concentrator (LSC). The relevant steady-state characteristics of eighteen common organic laser dyes are given. The relative spectral homogeneity of such dyes are shown to depend upon the surrounding material using narrowband laser excitation. We developed three independent techniques for measuring self-absorption rates; these are time-resolved emission, steady-state polarization anisotropy, and spectral convolution. Preliminary dye degradation and prototype efficiency measurements are included. Finally, we give simple relationships relating the efficiency and gain of an LSC to key spectroscopic parameters of its constituents.
\end{abstract}

\section{Introduction}

The luminescent solar concentrator (LSC) offers the promise of reducing the cost of photovoltaic energy conversion by the use of high gain concentrators which do not require tracking. The conceptual operation of the LSC is based on light pipe trapping of luminescence induced by the absorption of solar radiation. A transparent material, such as Plexiglas, is impregnated with guest luminescent absorbers such as organic dye molecules. Solar photons entering the upper face of the plate are absorbed, and photons are then emitted. Snell's law dictates that a large fraction of these luminescent photons will be trapped by total internal reflection; for example, $74 \%$ of an isotropic emission will be trapped in a PMMA plate having an index of refraction of 1.49. Successive reflections transport the luminescent photons to the edge of the plate where they can enter an edge-mounted array of solar cells.

We previously discussed in LSC- $1^{1}$ the primary issues governing the applicability of LSC devices; these issues are solar absorption bandwidth, self-absorption (or the reabsorption of emission from a particular dye or inorganic ion by another similar emitting center), and photodegradation. Our primary concern in this paper is to demonstrate experimental techniques and appropriate theoretical interpretations for determining the efficacy of a given dye in an LSC device. We will also

All authors are with California Institute of Technology, A. A. Noyes Laboratory of Chemical Physics, Pasadena, California 91125.

Received 8 June 1981.

0003-6935/81/213733-22\$00.50/0.

(C) 1981 Optical Society of America. investigate the effects of the relative spectral homogeneity, as determined by the type of substrate material used.

This paper is organized as follows: Section II describes spectroscopic techniques and results of measurements made on organic laser dyes. Such measurements include absorption, emission, and excitation spectra, relative spectral homogeneity in a variety of host materials, spatial filtering effects, and the polarization dependence of the output emission. We also utilize transient spectroscopic methods to find the observed lifetime of dye excitations as a function of concentration in the presence of self-absorption. Preliminary dye degradation data and prototype efficiencies are also described. Section III continues the treatment of our previous paper (LSC-1), extending the analysis of the self-absorption phenomenon to model the observed spectral shifts, the rate of depolarization of the output emission, and the lifetime of the emission with concentration. In Sec. IV we show how these results allow us to determine the efficiency of a single dye LSC plate as a function of the size or geometric gain of the plate. We conclude in Sec. V with our perceptions of the impact of the above developments on the potential utility of the LSC.

\section{Experimental Techniques and Results}

\section{A. Materials}

We restricted our study of luminescent centers to the organic laser dyes because of their high quantum efficiency of luminescence, their solubility in methyl methacrylate and organic solvents, and their ready availability. The source of the dyes was Exciton Chemical Co. ${ }^{2}$ Dyes were used as received without 
further purification. Liquid samples were made at known concentrations by dissolving quantities of dye weighed on a Cahn-25 electrobalance in reagent grade methanol. These samples were stored in soda-lime glass bottles and kept in darkness. The concentration of the samples for absorption measurements was chosen so that the peak optical density of a 1-cm path length of the solution between 10,000 and $30,000 \mathrm{~cm}^{-1}$ was between 0.5 and 1.5 .

Spectra were also taken of some of these dyes in a variety of other hosts. The principal solid matrix material was polymethyl methacrylate (PMMA), which usually contained $5 \%$ hydroxy ethyl methacrylate by weight to increase the solubility of the more polar dyes. Large plates $(1 \mathrm{~m}$ square $\times 0.3 \mathrm{~cm}$ thick $)$ were commercially made to our specifications by Acrilex Inc. ${ }^{3}$ Smaller test samples were fabricated in our laboratory in the following manner: Aldrich monomer, containing hydroquinone monomethyl ether as an inhibitor, was purified by fractional distillation in a nitrogen atmosphere using a vacuum-jacketed vigreaux column. This distilled monomer was combined with technical grade hydroxy ethyl methacrylate, and the desired dyes were dissolved therein. Small amounts of methanol and/or acetic acid were added to the solution to increase the dye solubility if rhodamine dyes were to be used. Some samples were prepared by adding the concentrated dye-monomer solution to prepolymerized PMMA and continuing the polymerization. However, best results were usually obtained by polymerizing just the monomer-dye solution. Two percent by weight of azobisisobutyronitrile was added as an initiator, and the mixture was poured into a mold formed by two glass plates. The plates were separated by a polyethylene tubing gasket and by aluminum spacers around the periphery to maintain a constant plate thickness. A very thin coat of silicon vacuum grease on the glass plates acted as mold release agent. These molds were

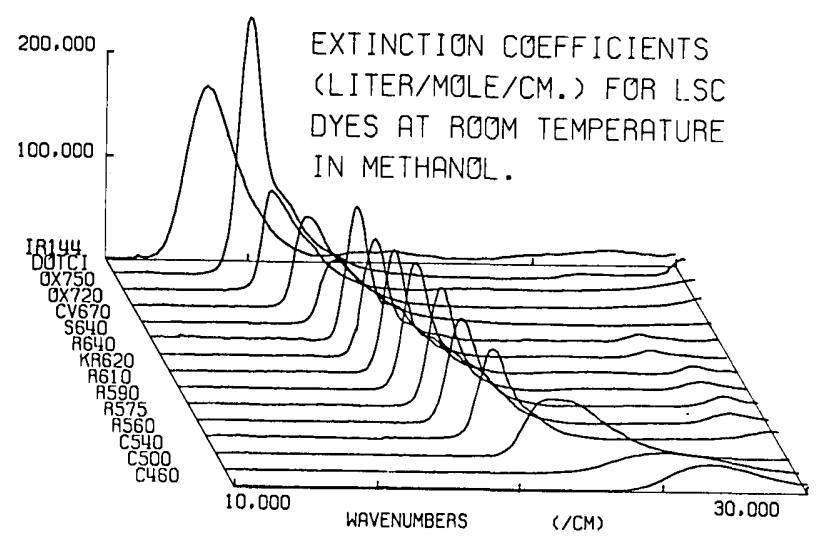

Fig. 1. Composite of extinction coefficient spectra for a variety of representative organic laser dyes. Vertical axis is extinction coefficient (liter $/ \mathrm{mole} / \mathrm{cm}$ ), and horizontal axis is in wave numbers. All spectra are from low concentration methanol solutions. then immersed in a water bath with an oil surface film and placed in a convection oven. Polymerization was initiated at $\sim 85^{\circ} \mathrm{C}$, when a noticeable increase in viscosity occurred, at which time the temperature was lowered to $55^{\circ}$ for $48 \mathrm{~h}$, followed by a final curing at $95^{\circ}$ for $4 \mathrm{~h}$. Typically, significant fractions of the dye did not go into solution, so that the dye concentrations in the final plates were assayed by measuring the peak optical density and assuming that the peak extinction coefficient was that of the methanol solutions. We found that dye concentrations in excess of $10 \mu \mathrm{M}$ caused significant amounts of monomer to remain unpolymerized in the cured plates in the case of rhodamine and oxazine dyes, and that this monomer could be slowly driven out by vacuum degassing at $50^{\circ} \mathrm{C}$. After curing, the plates were removed from the molds and were scribed and broken to size. The edges were polished with a sequence of grits; the final buffing compound was a cerium oxide rouge.

We also developed an alternative technique of doping the plastic which has the great advantages of not requiring distillation, casting, or curing. If a commercial transparent PMMA plate or rod is immersed in a methanol solvent containing the dye of interest, the dye will diffuse into the plate along with the methanol. A solution of $9 \%$ dichloromethane by volume in methanol was found to be the best compromise between speed of infusion and maintaining good surface finish on the plate. The time required to achieve useful dye concentrations in the plastic for a $20-\mathrm{cm}$ (8-in.) rod was $\sim 15$ $\mathrm{min}$, and for a $40-\mathrm{cm}$ (16-in.) plate the time was $\sim 12 \mathrm{~h}$, both at room temperature. Coumarin-540 infused faster than rhodamine-640, possibly due to the difference in molecular weight. It appeared to the eye that the dyes typically resided in a film between 0.1 and 1 $\mathrm{mm}$ from the surface of the plate, depending on temperature and soak time, so that the infusion technique does not yield a uniform dye concentration across the thickness of the plate. It is unlikely that the dye concentration in the film would be greater than that of the soaking solution, so that the solution concentration yields an upper bound on the local dye concentration. Measuring the peak optical density and assuming a uniform dye dispersion in the plate gives a lower limit on the concentration in the region of the plate where most of the dye reside.

\section{B. Steady-State Spectroscopy}

Absorption spectra of all samples were made using either a Cary-14 or a Cary-17 dual-beam spectrophotometer. These spectra and their associated base lines were digitized using a Houston Instruments Hipad digitizer. The digitized spectra were corrected for base line drift and stored on diskette using a Terak packaged PDP 11-03/2. Figure 1 shows a composite of a number of these absorption spectra plotted by a High Plot digital plotter on the same system. The digitization accuracy was five-hundredths of an inch, which is finer than the pen line of the chart recorder output. The base line noise in the spectra is typically 1000 liters/ mole/cm (SNR 100). 


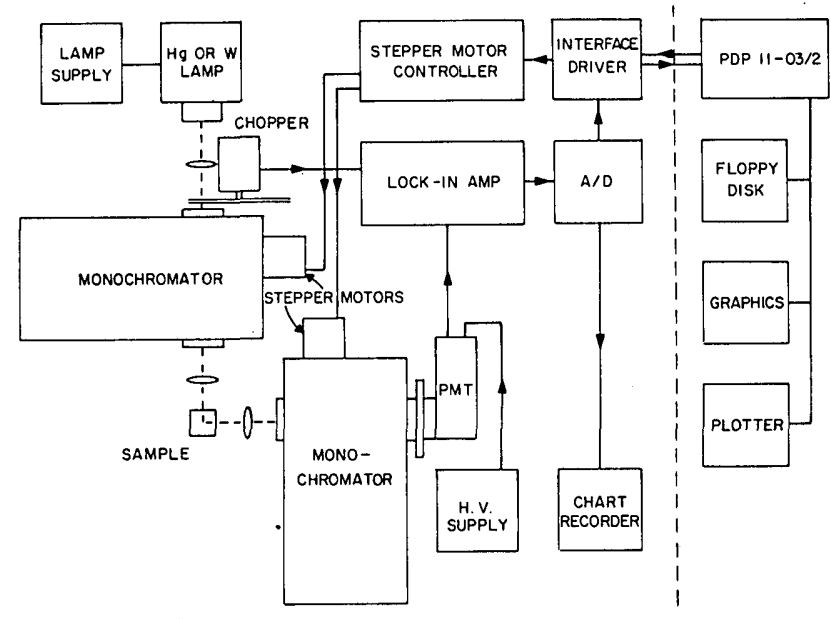

Fig. 2. Schematic of the apparatus for emission and excitation measurements. Regulated mercury or tungsten source was focused down, chopped, and monochromated prior to exciting the sample. Resulting emission was monochromated and detected by a PMT, and the signal was amplified with phase-sensitive detection. Remote computer controlled both monochromators and recorded the measured spectra.

Emission and excitation spectra of the dyes were made at micromolar concentrations using a computercontrolled apparatus, as shown in Fig. 2. The excitation source was either a 200-W Oriel 6323 tungsten lamp or a $200-W$ Oriel 6137 high pressure mercury lamp. The light was collimated with quartz optics, chopped by a PAR 191 chopper, and monochromated by a Jarrell-Ash (model 82-410, $f / 3.5,0.25-\mathrm{m}$ ) monochromator with either a 6000 - $\AA$ blazed grating with 1180 grooves $/ \mathrm{mm}$ or a 3000 - $\AA$ blazed grating with 2365 grooves $/ \mathrm{mm}$. For the phase sensitive detection to produce accurate excitation and emission spectra when chopping the excitation source, the lifetimes of the dyes must be short compared with the chopping period $(2.5 \mathrm{msec}$ in these experiments). This condition was fulfilled in all these measurements. The excitation linewidth was fixed at $90 \AA$ across the tuning range.

The emission $90^{\circ}$ from the excitation was analyzed by a similar Jarrell-Ash monochromator, in this case with an adjustable resolution of $2-90 \AA$. 'The output light was detected by a 928 Hamamatsu PMT biased at $900 \mathrm{~V}$; the PMT output was terminated by $100 \mathrm{k} \Omega$ parallel with a PAR-HR8 lock-in amplifier. The analog output of the lock-in was digitized recorded by the PDP 11-03/2. Care was taken to keep the PMT current at least $20 \%$ below its rated output current of $100 \mu \mathrm{A}$. The $\mathrm{RC}$ time constant of the lock-in was kept at least as short as the time between digital sampling of the emission. These samples were taken typically every 10 $\AA$. Both monochromators were driven by a Slo-Syn SP151 driver so that either one or both monochromators could scan under the control of the PDP 11-03/2. This allowed acquiring spectra in $\mathrm{cm}^{-1}$ even though the monochromators had wavelength drives. Computer control also provided automatic backlash correction. Frequency calibration of the system was done with the
$5451-\AA$ line from a mercury germicidal lamp, and the system response was measured using an Eppley Laboratory calibrated EPI-1669 quartz halogen lamp in a custom-made housing. The output of the HR-8 was digitized in a 12-bit analog-to-digital converter built around the Intersil ICL-7107. This generated a parallel digital input to the remote PDP 11-03/2. The system response function was stored on diskette; this was determined to be the response of the system to the calibrated tungsten lamp divided by the known spectrum of the tungsten lamp. Emission spectra were corrected by dividing out this system response and were normalized so that the luminescence integrated over all wave numbers equals 1 . The peak position of the emission spectra for the dyes tested is given in Table I. Correcting the excitation spectra for the response of the system requires a calibrated detector as well as a calibrated source. Using both it is possible to determine the response of the monochromator that is scanning the excitation. We made the approximation that our PMT response.was flat over the region of interest. In this approximation, the variation of the intensity of the scanning excitation is the measured response to the calibrated tungsten lamp. Excitation spectra were corrected by dividing out this measured response.

Both the emission and excitation spectra of these dyes were taken only at micromolar dye concentration to minimize the red-shifting effect of self-absorption. The optical density of the sample in the region where the absorption and emission overlap must be sufficiently low that the blue tail of the emission spectrum is not artificially filtered out. The excitation samples must be dilute for a second reason. Away from the peak of the absorption, where the absorption per unit length is small, the exciting beam intensity does not vary greatly along the beam length in the sample. However, as the excitation is scanned across the peak of the absorption, most of the exciting light is absorbed in a thin surface layer in the sample if the concentration is too high. This effect changes the spatial distribution of the emission and alters the observed emission spectrum.

Measurements were also made on the spectral characteristics of multiple dye solutions. Dyes could not be combined indiscriminately, possibly due to agglomeration phenomenon between some dyes which quenched the emission when they were in solution together. For example, our oxazine- 720 or oxazine- 750 solutions showed quenching of their emission when they were in a solution with rhodamine- 610 or rhodamine-590. An example of a successful multiple dye combination is sulforhodamine- 640 , rhodamine-590, and coumarin540. In Figures 3 and 4 we show emission and excitation spectra, respectively, for an oxazine-720 methanol solution and for an oxazine-720-rhodamine-640-coumarin-540 methanol mixture at micromolar and hundred micromolar concentrations. The emission spectra of the multiple-dye solution at low concentration are dominated by rhodamine and coumarine emission, while at high concentration the oxazine dominates the emission. If we detect the emission intensity of oxazine, as shown in Fig. 4, as a function of excitation energy, the 
Table I. Optical Properties of Dyes used in the LSCs

\begin{tabular}{|c|c|c|c|c|c|c|c|c|}
\hline $\begin{array}{c}\text { Exciton Dye } \\
\text { (Kodak name) }\end{array}$ & $\begin{array}{c}\epsilon_{\max } \\
\begin{array}{c}\text { (mole } / \mathrm{cm} / \text { liter } \\
\text { in methanol) }\end{array}\end{array}$ & $\begin{array}{c}\bar{\nu}\left(\epsilon_{\max }\right) \\
\mathrm{cm}^{-1}\end{array}$ & 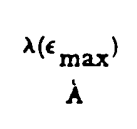 & $\begin{array}{c}\bar{\nu}\left(f_{\max }\right) \\
\mathbf{c m}^{-1}\end{array}$ & $\begin{array}{c}\lambda\left(f_{\max }\right) \\
\grave{\lambda}\end{array}$ & $\begin{array}{l}\text { Stoke Shift } \\
\nu\left(\epsilon_{\max }\right)-\nu\left(f_{\max }\right) \\
\mathrm{cm}^{-1}\end{array}$ & $\begin{array}{c}\eta \\
\text { Solvent }\end{array}$ & $\begin{array}{l}\text { CODE } \\
\pm 30_{\kappa}^{c}\end{array}$ \\
\hline $\begin{array}{l}\text { Coumarin-480 } \\
\text { (Coumarin-102) }\end{array}$ & 22,000 & 25,730 & 3,890 & 21,200 & 1,720 & 4,560 & $\begin{array}{c}0.58^{\mathrm{a}} \\
\text { ethanol }\end{array}$ & 110. \\
\hline Coumarin-500 & 19,900 & 25,660 & 3,900 & 20,200 & 4.950 & 5,440 & $\begin{array}{c}0.53^{2} \\
\text { ethanol }\end{array}$ & 140. \\
\hline $\begin{array}{l}\text { Coumarin-535 } \\
\text { (Coumarin-7) }\end{array}$ & 52,200 & 23,120 & 4,320 & 20,400 & 4,900 & 2,720 & & 90. \\
\hline $\begin{array}{l}\text { Coumarin-540 } \\
\text { (Coumarin-6) }\end{array}$ & 52,200 & 21,860 & 4,580 & 19,700 & 5,070 & 2,130 & $\begin{array}{c}0.78^{a} \\
\text { ethanol }\end{array}$ & 80. \\
\hline $\mathrm{DCM}$ & 28,900 & 21,500 & 4,650 & 15,700 & 6,360 & 5,760 & $\begin{array}{c}0.71^{b} \\
\text { DMSO }\end{array}$ & 240 \\
\hline $\begin{array}{l}\text { Rhodamine-560 } \\
\text { (Rhodamine-110) }\end{array}$ & 82,000 & 20,120 & 4,970 & 19,000 & 5,250 & 1,080 & $\begin{array}{l}0.85^{c} \\
\text { ethanol }\end{array}$ & 25. \\
\hline Rhodamine-575 & 93,800 & 19,330 & 5,170 & 18,300 & 5,460 & 1,010 & & 35. \\
\hline $\begin{array}{l}\text { Rhodamine-590 } \\
\text { (Rhodamine-6G) }\end{array}$ & 107,000 & 18,940 & 5,280 & 18,000 & 5,550 & 900 & $\begin{array}{l}0.98^{\mathrm{d}} \\
\text { methanol }\end{array}$ & 25. \\
\hline $\begin{array}{l}\text { Rhodamine-610 } \\
\text { (Rhodamine-B) }\end{array}$ & 114,000 & 18,380 & 5,440 & 17,500 & 5,710 & 870 & $\begin{array}{l}0.5^{e} \\
\text { ethanol }\end{array}$ & 36. \\
\hline $\begin{array}{l}\text { Kiton red }-620 \\
\text { (Sulforhodamine-B) }\end{array}$ & 111,000 & 17,990 & 5,560 & 17,300 & 5,800 & 740 & $\begin{array}{c}0.83^{8} \\
\text { ethanol }\end{array}$ & 16. \\
\hline $\begin{array}{l}\text { Rhodamine- } 640 \\
\text { (Rhodamine-101) }\end{array}$ & 106,000 & 17,670 & 5,660 & 16,800 & 5,940 & 830 & ethanol $^{\text {c }}$ & 17. \\
\hline $\begin{array}{l}\text { Sulforhodamine-640 } \\
\text { (Sulforhodamine-101) }\end{array}$ & 120, onn & 17,360 & 5,760 & 16,700 & 6,000 & 690 & $\frac{1}{\text { ethanol }}$ & 17. \\
\hline $\begin{array}{l}\text { Cresile violet }-670 \\
\text { (Oxazine-9) }\end{array}$ & 57,900 & 16,880 & 5,920 & 16,100 & 6,220 & 810 & ${ }_{\text {methanol }}^{0.54^{f}}$ & 17. \\
\hline $\begin{array}{l}\text { Oxazine-720 } \\
\text { (Oxazine-170) }\end{array}$ & 81,800 & 16,170 & 6,190 & 15,600 & 6,420 & 600 & & 17. \\
\hline Oxazine- 750 & 90,600 & 15,140 & 6,600 & 14,500 & 6,920 & 680 & & 25. \\
\hline DODCI & 238,000 & 17,160 & 5,830 & 16,500 & 6,050 & 630 & & 11. \\
\hline DOTCI & 236,000 & 14,770 & 6,770 & 14,000 & 7,140 & 770 & & 8. \\
\hline IR-144 & 153,000 & 13,560 & 7,370 & 12,000 & 8,340 & 1,560 & & 16. \\
\hline
\end{tabular}

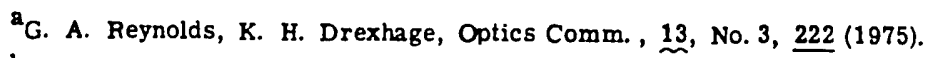

P. R. Hammond, Optics Comm., 29, No. 3, 331 (1979).

ck. H. Drexhage, 'Structure and Properties of Laser Dyes, "ed. F. P. Schafer, Topics in Dye Lasers, Applied Physics I (Springer New York, 1977) p. 144.

dA. Baczynskf, T. Marszalek, H. Walerys, B. Zietek, Acta Phys. Polo., A44, No. 6, $\underline{805}$ (1973).

T. Karstens, K. Kobs, J. Phys. Chem., 84, No. 14, 1871 (1980).

D. Magde, J. H. Brannon, T. L. Cremers, J. Olmsted II, J. Phys. Chem., 83, No. 6, 696 (1979).

B. M. Drake, R. T. Morse, R. N. Steppl, D. Young, Chem. Phys. Lett., 35, No. 2, 181 (1975).

hC. F. Rapp et al., Final Report of Owens Illinols, Sand77-7005, p. 40. 


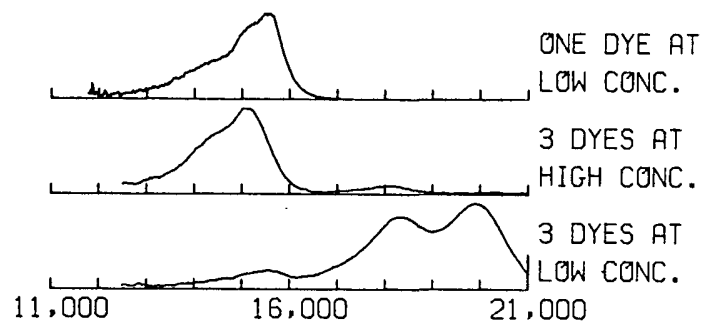

WAVENUMBERS

Fig. 3. Three emission spectra of methanol dye solutions resulting from 22,220-wave number (4500- $\AA$ ) excitation. Top spectrum is from a micromolar oxazine-720 solution, and the bottom two are micromolar and hundred micromolar solutions, respectively, of coumarin-540, rhodamine- 640 , and oxazine- 720 .

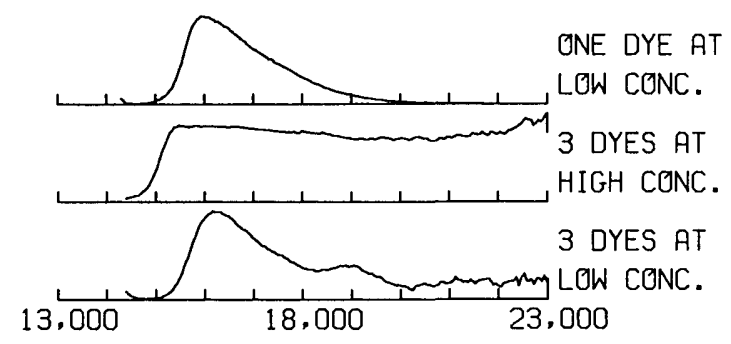

WAVENUMBERS

Fig. 4. Three excitation spectra of methanol dye solutions due to 15,630-wave number $(6400-\AA)$ detection. Three spectra correspond to the same three solutions described in Fig. 3.

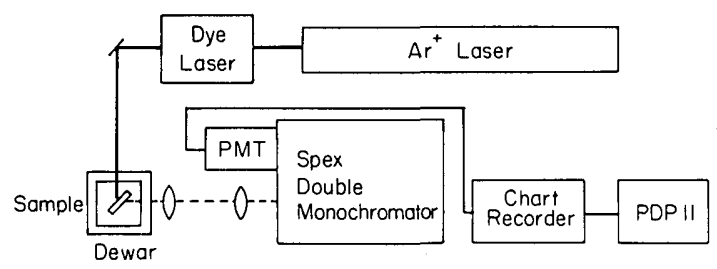

Fig. 5. Diagram of the apparatus used to measure the luminescence spectra of liquid, cast plastic, and diffused plastic samples as a function of excitation energy and temperature. Either the argon-ion laser or the tunable dye laser was used as the excitation source. Apparent sample temperature was maintained at either 77 or $300 \mathrm{~K}$. Resulting emission was analyzed by a Spex 14018 double monochromator.

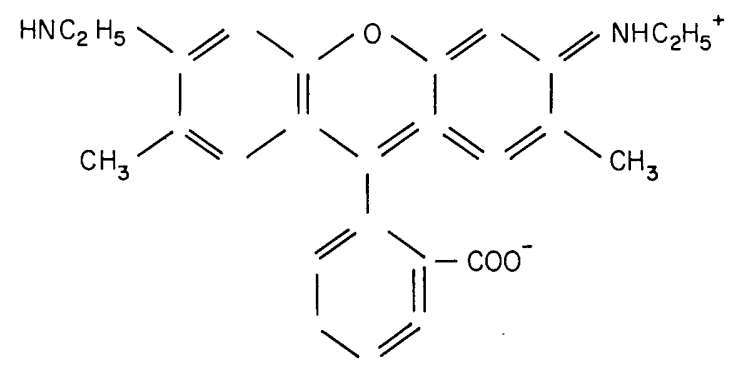

Fig. 6. Structure of rhodamine-575. low concentration multiple dye solution is dominated by the pure oxazine absorption peak, while the high concentration multiple dye solution gives a nearly constant response across the visible spectrum.

\section{Spectral Inhomogeneity}

A primary objective of our work was determination of the homogeneity of the absorption and emission spectra in solution, in cast plastic, and in diffused plastic and to determine the temperature dependence of the anti-Stokes shifted emission. We shall call this the spectral inhomogeneity of the dye in these various cases. To achieve these objectives the luminescence spectra were taken using the apparatus shown in Fig. 5. A Spectra-Physics 160 argon-ion laser was used directly as the exciting source or served as a pump for a Spectra-Physics $375 \mathrm{~A}$ dye laser. The dye laser operated with a glycol solution of rhodamine-590. When we excited the sample at an energy considerably below its peak absorption, it was important that very little spontaneous emission of a higher energy than the principal lasing wavelength be present in the sample excitation beam. The dye laser was therefore followed by several sharp cut filters, a dispersive prism, and a slit aperture. Neutral density filters were used to adjust the input power to the sample. The samples were held in a glass Dewar with windows positioned for both the excitation beam and for the resulting right-angle emission. The luminescence spectra were taken both at room temperature and with the samples immersed in liquid nitrogen. Since the excitation was not chopped, it was important to eliminate stray room light as a source of excitation. The luminescence resulting from the laser excitation was collected by a Spex 1419A sample illuminator and then analyzed by a Spex 14018 double monochromator with 2400 -groove/mm holographic gratings. The sample illuminator was a collection optics stage, originally designed for Raman spectroscopy, which contained the dispersive prism, slit, sample translation stage, and matched $f /$ No. optics for the spectrometer. A polarizing filter in the collection optics was used to suppress scattered laser light, followed by a depolarizing prism to compensate for the polarization response of the gratings. The spectrally resolved output was measured by a Hamamatsu 955 photomultiplier and a Spex DPC-2 photon counter. The final chart recorder output was digitized and stored in the PDP 11-03/2. System response correction was effected by digitizing the measured spectrum of a calibrated tungsten lamp, as described in the previous section.

Rhodamine- 575 was chosen for study because it can be excited on the extreme red edge of its absorption band by the rhodamine-590 dye laser. The structure of rhodamine-575 shown in Fig. 6 is also very similar to other xanthene dyes, so that the results of these measurements should be representative of most of that class of laser dyes. The liquid sample studied in this manner was $18-\mu \mathrm{M}$ rhodamine- 575 in methanol. This was mounted in a 1.3-mm o.d. capillary tube, oriented so as to minimize the path length of the output emission. 

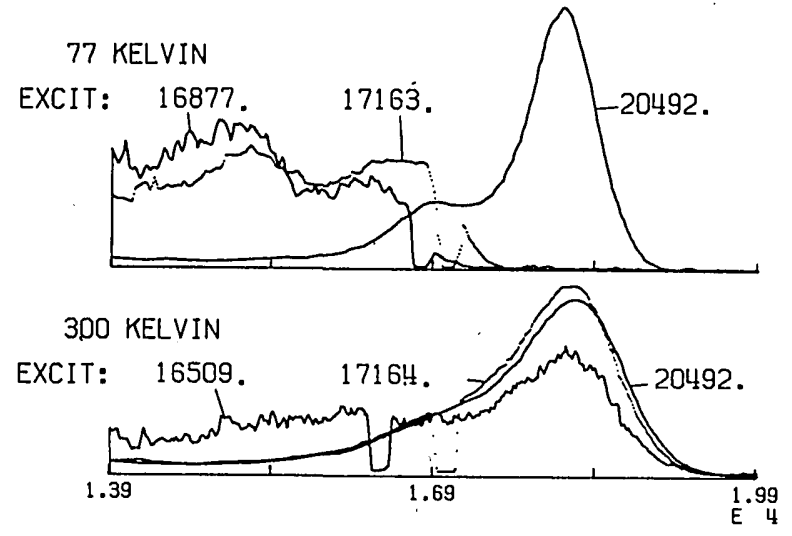

WAVENUMBERS

Fig. 7. Emission spectra of rhodamine-575. Lower three plots show the room temperature emission of rhodamine- 575 in methanol due to excitation at $16,509,17,164$, and 20,492 wave numbers $(6075,5826$, and $4880 \AA$, respectively). Upper three plots show similar emission spectra taken at $77 \mathrm{~K}$ using excitations at $16,877,17,163$, and 20,492 wave numbers (5925, 5826, and $4880 \AA$, respectively). Room temperature spectra show that the dye emits with essentially the same spectrum for all the excitation energies.

When the methanol is cooled to liquid nitrogen temperatures it forms a fractured glass. The cast plastic sample was a 20- $\times 25-\mathrm{mm}$ PMMA rod containing an effective concentration of $14-\mu \mathrm{M}$ rhodamine- 575 . The diffused sample was a $1.6-\mathrm{mm}$ diam rod of Rohm \& Haas Plexiglas that had been soaked for $4 \mathrm{~h}$ in a $100-\mu \mathrm{M}$ solution of rhodamine- 575 in a mixture of 0.91 methanol and 0.09 dichloromethane by volume.

The results from the liquid sample are shown in Fig. 7. The $200-\mathrm{cm}^{-1}$ wide notches in the spectra are due to a shutter which blocks the photomultiplier tube as the spectrometer is scanning across the laser frequency. The lower three spectra correspond to the room temperature emission for laser excitations at 16,509, 17,164, and $20,492 \mathrm{~cm}^{-1}(6057,5826$, and $4880 \AA$, respectively). Note that the peak of the emission for the lowest energy excitation is at the same frequency as that of the highest energy excitation, despite the fact that some of the emitted photons were blue-shifted as much as 1700 $\mathrm{cm}^{-1}$ up from the excitation frequency. This antiStokes shift in energy is $>5 \mathrm{kT}$ for room temperature spectra. Introducing sharp-cut filters into the excitation beam centered at the lasing wavelength caused a reduction in the overall emission intensity in proportion to the reduction in the excitation intensity but caused no shift in the observed spectral shape. We concluded that these emission spectra did not include stray excitation light of higher energy. The upper plot shows emission spectra at $77 \mathrm{~K}$ due to excitations at 16,877 , 17,163 , and $20,492 \mathrm{~cm}^{-1}(5925,5826$, and $4880 \AA$, respectively). The luminescence spectrum due to the highest energy excitation is a slightly narrower version of the room temperature spectrum. The low energy excitation at low temperature produces negligible emission at higher energies, which agrees with the previous result that the emission at room temperature from
77 KELVIN

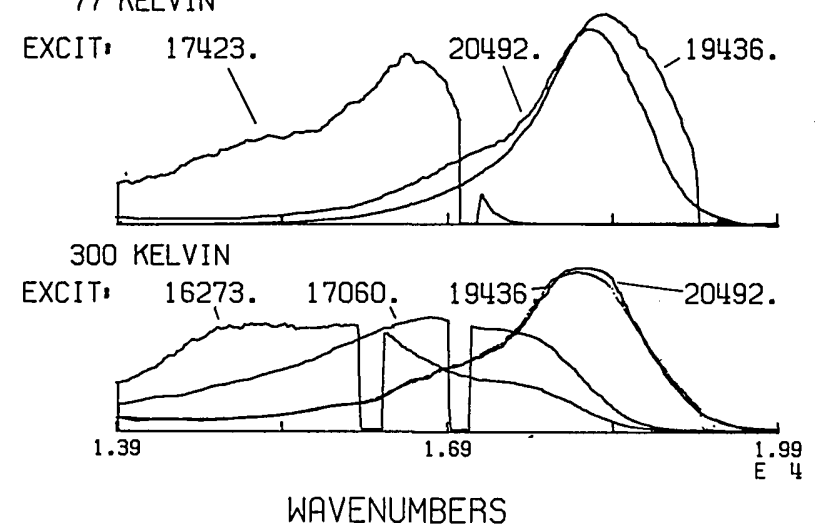

Fig. 8. Emission spectra of rhodamine-575. Lower three plots show the luminescence of rhodamine-575 cast in PMMA at room temperature for excitations at $16,273,17,060,19,436$, and 20,492 wave numbers $(6145,5826,6145$, and $4880 \AA$, respectively). Notice the pronounced skewing of the emission toward lower energies by the low energy excitations. Upper plot shows luminescence at $77 \mathrm{~K}$ for excitations at $17,423,19,436$, and 20,492 wave numbers $(5740,5145$, and $4880 \AA$, respectively).

low energy excitation was due to anti-Stokes shifting of the excitation.

Figure 8 gives corresponding results for rhodamine575 cast in PMMA. The lower plot shows the room temperature luminescence spectra normalized to unit area. These spectra differ from the methanol solutions in that the emissions produced by low energy excitations at 16,273 and $17,060 \mathrm{~cm}^{-1}$ (6145 and $5862 \AA$ ) are greatly skewed toward the red, whereas the higher energy excitations as 19,436 and $20,492 \mathrm{~cm}^{-1}$ (5145 and $4880 \AA$ ). produce emission with the same general shape and position as the methanol room temperature emission. The upper plot shows the low temperature emission due to excitations at $17,423,19,436$, and $20,948 \mathrm{~cm}^{-1}(5740$, 5145 , and $4880 \AA$, respectively). As in the liquid samples, the anti-Stokes shifted emission is suppressed at $77 \mathrm{~K}$. However, the emission shape is still dependent on the position of the excitation.

The diffused plastic sample shows anti-Stokes behavior intermediate between the cast plastic and the liquid solution. Figure 9 shows the room temperature

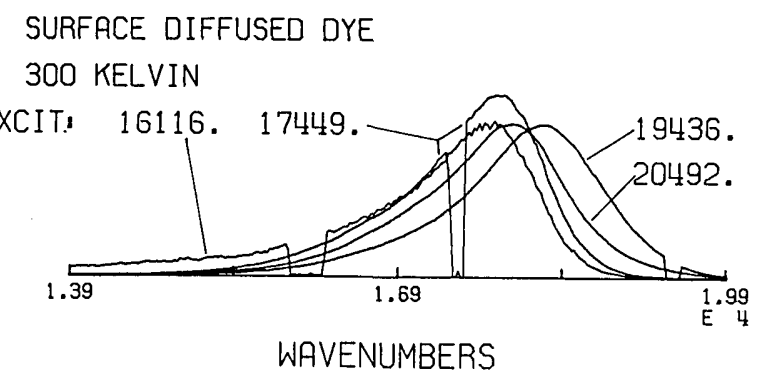

Fig. 9. Emission of rhodamine-575 diffused into PMMA. Emission spectra were taken at room temperature. Excitation energies were $16,116,17,449,19,436$, and 20,492 wave numbers $(6205,5731,5145$, and $4880 \AA$, respectively). 


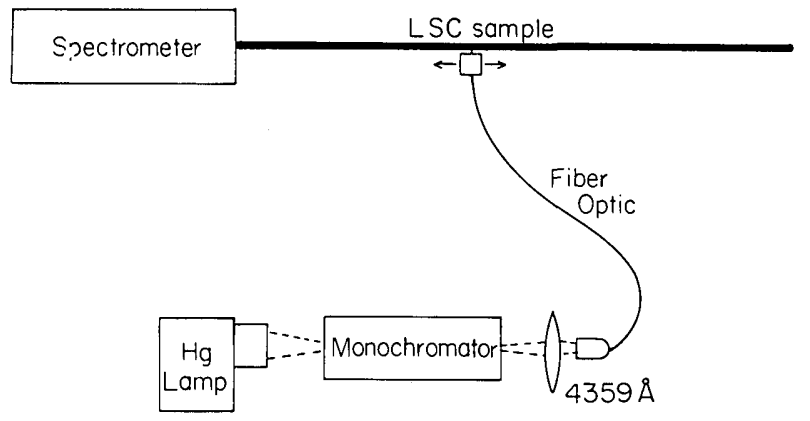

Fig. 10. Apparatus for measuring the intensity and spectral shifts of the sample emission as a function of the path length through the sample traveled by the emission. The 22,940 -wave number (4359- $\AA$ ) line from a mercury lamp was monochromated, chopped, and focused onto an optical fiber. The opposite end of the fiber was scanned horizontally with a micrometer stage along the rod-shaped sample of LSC material. Output was detected by a scanning monochromator.

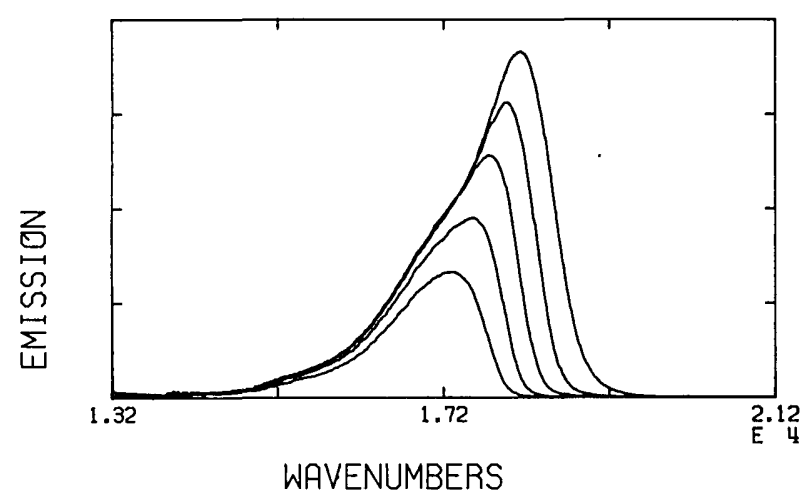

Fig. 11. Emission spectra sequence from a $92-\mu \mathrm{M}$ methanol solution of rhodamine-575. Sample path lengths for each spectrum in order of the most to least intense were $0.3,1.0,3.0,10.0$, and $30.0 \mathrm{~cm}$. Spectra have been corrected for the system response of the detection monochromator and the PMT, so that the amplitude of each spectrum corresponds to the intensities emerging from the end of the rod.

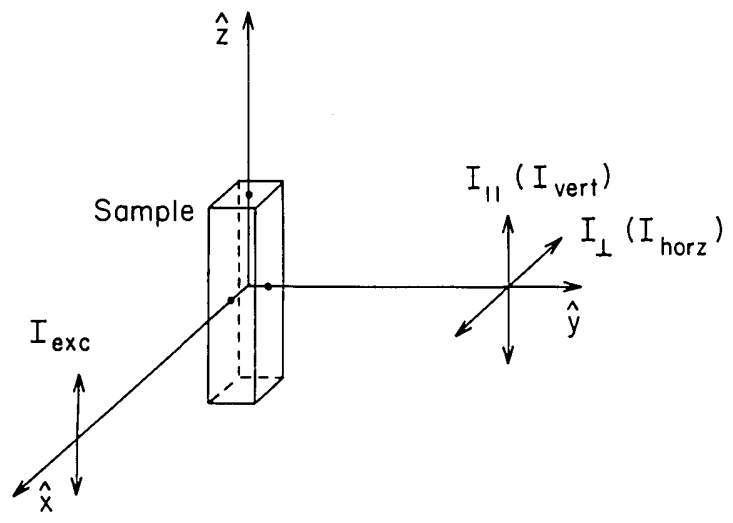

Fig. 12. Principal direction for polarization anisotropy measurements. Monochromated light excites rhodamine- 575 in an ethylene glycol solution, and the resulting emission is detected $90^{\circ}$ to the axis of excitation. Excitation is polarized with its electric field either horizontal or vertical, and the emission is similarly polarized horizontally or vertically (perpendicular or parallel, respectively). emission of a PMMA sample soaked in a methanol solution of rhodamine-575. The excitation positions are $16,116,17,449,19,436$, and $20,492 \mathrm{~cm}^{-1}(6205,5731$, 5145 , and $4880 \AA$, respectively). The emission spectra have been normalized to unit area. While there is some variation of the peak position with excitation energy, we find that the spectra are dominated by large antiStokes shifts for low excitation energies, as was the case for the methanol solutions.

\section{Spatial Filtering Effects}

We measured the changes in the intensity and spatial characteristics of dye emission by scanning the position of a small excitation spot with respect to the sample and then observing the resulting emission from a fixed point on the sample. Preliminary results from such an experiment were given in LSC-1. Figure 10 depicts the apparatus used. The $22,940-\mathrm{cm}^{-1}(4359-\AA)$ radiation from a $200-W$ Oriel 6137 high pressure mercury lamp was filtered by a Bausch \& Lomb 33-86-25 monochromator, modulated with a PAR-BZ-1 chopper, and focused by a $\times 40$ microscope objective onto one end of a 1-m section of a Math Associates OC-1200 glass optical fiber bundle. Since we were interested in the change of intensity as well as spectral shape of the emission with increasing path length through the sample, it was important that the position of the excitation source at the sample be adjustable without any variation in intensity. Small changes in the curvature of an optical fiber cause little change in its transmission, so that the free end of the fiber was used as the mobile excitation. The free end was mounted on a micrometer stage that would track parallel to the rod-shaped sample of LSC material, as shown in Fig. 10. Three different types of samples were tested: methanol solutions; cast PMMA; and diffused PMMA. Samples were mounted inside 7-mm o.d. (5-mm i.d.) borosilicate glass tubes to maintain a uniform contact between the excitation fiber and the sample. The end of the tube adjacent to the detecting monochromator was sealed with a 1 -cm glass plug. The detection system was a 0.25 -m Jarrell-Ash spectrometer, a Hamamatsu 928 PMT, and a PAR HR-8 lock-in amplifier. The detection monochromator was scanned, and the output of the lock-in amplifier was sampled by the PDP $11-03 / 2$, as described in the previous section.

The results from one such measurement are shown in Fig. 11. In this case the borosillicate glass tube was filled with a $92-\mu \mathrm{M}$ solution of rhodamine-575 in methanol. Spectra were taken for the following distances between the fiber excitation and the glass plug seal at the end of the tube: $0.3 ; 1.0 ; 3.0 ; 10.0$; and 30.0 $\mathrm{cm}$. System response corrections were made to compensate for the detection monochromator and PMT, as described in the previous section. Apart from this correction, the amplitudes of each spectrum correspond to the actual intensities emerging from the end of the sample rod. The predominant observed effect was that the higher energy portion of the band gradually disappears. 


\section{E. Steady-State Polarization Measurements}

We measured the relative polarization of emission from a polarized excitation. Light from a tungsten lamp was monochromated at $20,200 \mathrm{~cm}^{-1}(4950 \AA)$, chopped, and passed through a polarizer (Polaroid HN38). This light was focused onto an ethylene glycol solution of rhodamine-575. The concentration was varied during the course of the experiment by changing the dye solution. The resulting emission $90^{\circ}$ from the axis of the excitation was passed through a second polarizer, and the intensity at $17,860 \mathrm{~cm}^{-1}(5600 \AA)$ was measured with a second monochromator, a PMT, and a lock-in amplifier. The lamp, the monochromators, the chopper, the PMT, and the lock-in have been described previously. Figure 12 shows a schematic of the sample cell with the various combinations of light polarizations of the excitation and the emission.

Ethylene glycol was chosen because it has a viscosity of $\sim 20 \mathrm{cP}$ at room temperature and should produce a rotational diffusion time of the dye long enough that emission from a polarized excitation would also be partially polarized. If this is the case, emission polarized in the $Z$ direction should be more intense than emission polarized in the $X$ direction for a $Z$-polarized excitation (Fig. 12). $Y$-polarized excitation should produce equal intensities for both polarizations of emission. ${ }^{4}$ The expected polarization intensities are discussed in the theory section and in Appendix B. Dye solutions were used in place of cast plates to facilitate changing the dye concentrations.

We varied the dye concentration of the sample and measured the intensity of the $Z$ - and $X$-polarized emissions at each concentration. The sample dye concentrations were varied from 0.2 to $100 \mu \mathrm{M}$ by factors of 2. The path length in the sample traversed by the emission en route to the detection monochromator was $0.7 \mathrm{~cm}$. The observed intensities were corrected for background and the polarization response of the system.

Figure 13 shows the variation between the $Z$ - and $X$-polarized emissions for $Z$-polarized excitation as a function of concentration. The plotted value is the polarization anisotropy, which is the emission intensity of the $Z$ minus the $X$-polarized emission intensities, both divided by the sum of the $Z$ plus two times the $X$-polarized emission intensities. For very low concentrations, the anisotropy plateaus at $\sim 0.18$ or equivalently the ratio of the parallel to the perpendicular emissions are 1.65 . As the concentration increases, the apparent polarization of the emission decreases. The error bars reflect variations of repetitive measurements at a single concentration.

\section{F. Transient Emission Measurements}

We measured the time evolution of the emission intensity resulting from very short pulse excitation of dyes in a variety of hosts. Time-resolved fluorescence measurements were performed with a mode-locked argon-ion laser (Spectra-Physics model 171/342) and a single photon counting apparatus described recently by Robbins et al. ${ }^{5}$ of this laboratory. Samples were

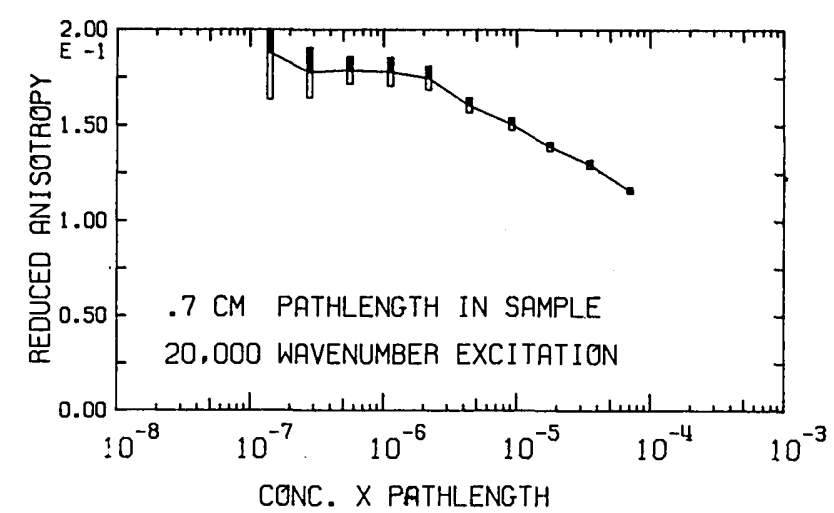

Fig. 13. Reduced polarization anisotropy vs sample concentration. Polarization anisotropy of the emission from rhodamine- 575 in ethylene glycol as a function of concentration for parallel polarized excitation is plotted. Reduced polarization anisotropy is defined to be the difference between the parallel and perpendicular emission intensities divided by the sum of the parallel and twice the perpendicular intensities. Solid line connects data points.

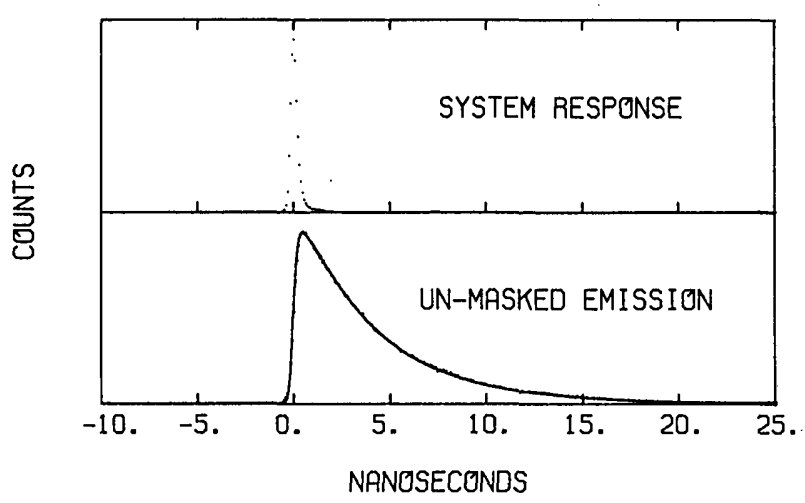

Fig. 14. System response and transient lifetime measurement. Upper plot shows a typical histogram of the system response to a $5145-\AA$ pulse from a mode-locked argon-ion laser with a duration of $<200$ psec scattered off of a dilute coffee creamer solution. Width and symmetry of the response were predominantly due to the PMT response. Lower plot shows the observed emission of a 4.6- $\mu \mathrm{M}$ rhodamine- 575 methanol solution superimposed on a best fit single exponential convoluted with the system response. Exponential fit gives a lifetime of 4.1 nsec.

illuminated with vertically polarized $19,436-\mathrm{cm}^{-1}$ (5145- $\AA$ ) light pulses at a repetition rate of $\sim 100 \mathrm{kHz}$ with a typical pulse halfwidth of $<200$ psec. Emission from the sample was monitored by a Philips XP2020Q photomultiplier. Cutoff filters at 5000 and $5700 \AA$ to eliminate scattered laser light, plus an analyzing polarizer to sample different emission polarization intensities, were placed between the sample and the detector when luminescent samples were measured. The system response, measured by laser light scattered from a dilute coffee creamer solution sample, is shown in the upper plot in Fig. 14 and usually had a response time of $<250$ psec. 


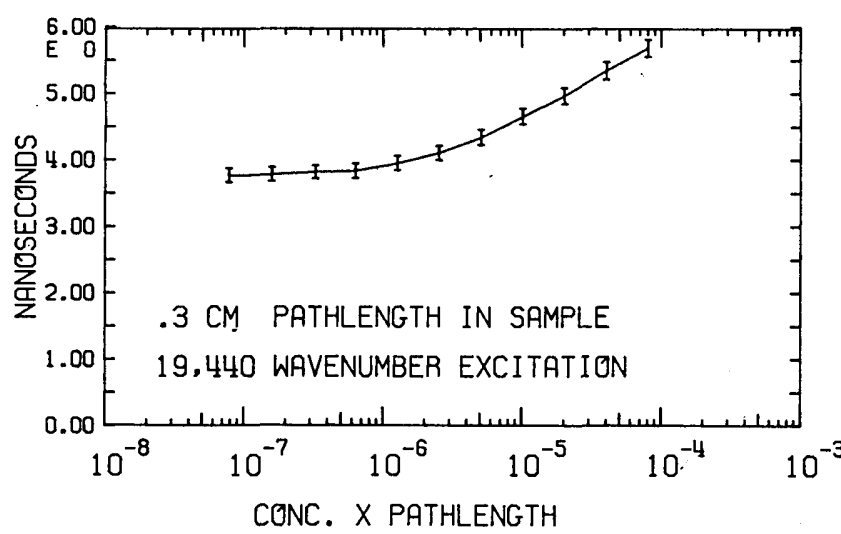

Fig. 15. Measured lifetime of rhodamine-575 in methanol as a function of dye concentration is plotted. Solid line serves to connect data points.

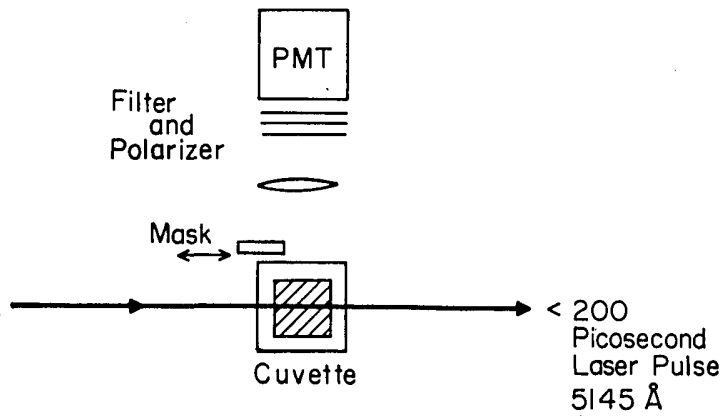

Fig. 16. Position of the first generation emission mask. The $5145-\AA$ laser excitation is $99 \%$ absorbed in the first $0.05 \mathrm{~cm}$ of the $460-\mu \mathrm{M}$ rhodamine- 575 methanol solution. Blocking just the leftmost portion of the cell, as shown, prevents the emission due to the initial excitation from being directly detected.

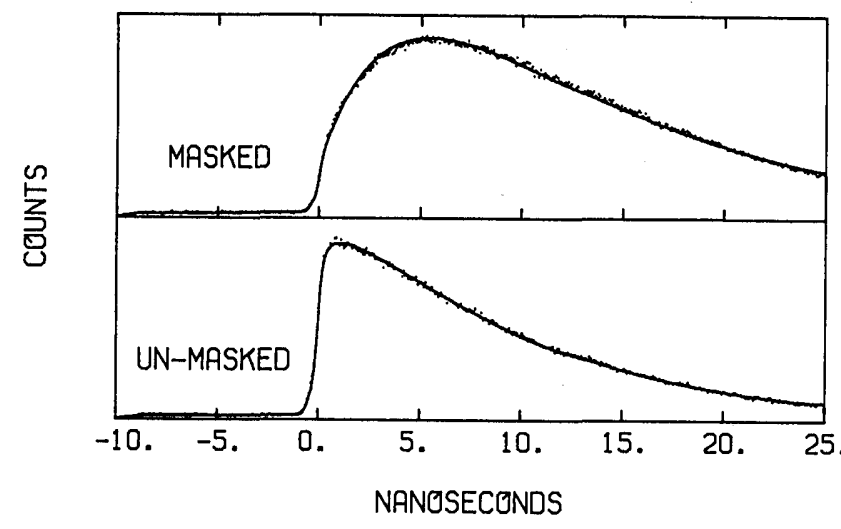

Fig. 17. Unmasked and masked transient emission measurements. Upper plot shows the transient emission spectrum from $460-\mu \mathrm{M}$ rhodamine- 575 methanol solution for the case where a mask is positioned between the region of the sample excited by the laser and detector. When the mask is removed, we obtain the spectrum shown in the lower half of the figure.
The first type of measurement we made was to find the total lifetime of the dye as a function of concentration. In this case the dye emission was filtered by a polarizer at $54.7^{\circ}$ from the laser's original vertical polarization to average between the emission of the parallel and perpendicular polarization components. The lower plot in Fig. 14 shows typical transient emission data for a $4.6-\mu \mathrm{M}$ solution of rhodamine- 575 in methanol. The dye was contained in a $1.0-\times 0.5-\mathrm{cm}$ cuvette, so that on average this emission passed through $0.5 \mathrm{~cm}$ of the sample before arriving at the collection optics. The extinction coefficient for rhodamine- 575 at 5145 $\AA$ was $\sim 90,000$ moles $/ \mathrm{cm}$ liter, so that, even at this low concentration, $\sim 40 \%$ of the laser excitation was absorbed by the sample. Superimposed on the raw data in Fig. 14 is a best fit convolution of the system response in the upper plot and a single exponential. The best exponential fit gave an observed lifetime of $4.13 \mathrm{nsec}$ at this concentration.

We measured apparent lifetimes for a sequence of dye concentrations from 0.2 to $100 \mu \mathrm{M}$ for rhodamine- 575 in both methanol and ethylene glycol. Figure 15 shows the fitted lifetimes as a function of concentration in methanol. The highest concentration used was a factor of 10 below the critical concentration for nonradiative transfer among similar dye molecules as defined by Förster. At low concentrations, the lifetime of rhodamine-575 approached an asymptotic value of $3.7 \mathrm{nsec}$, while at high concentrations it increased to three times its low concentration value. The error bars indicate the uncertainty of the lifetime in the numerical fitting of the data. As in the previous case, the emission was filtered with a polarizer $54.7^{\circ}$ from the vertical to sample equally the parallel and perpendicular polarizations. The emission traveled through $\sim 0.5 \mathrm{~cm}$ of sample before being detected, and care was taken not to mask any portion of the emission from the cell from illuminating the PMT.

To help determine the mechanism for the lifetime lengthening of the dye with increasing concentration, we performed a measurement with part of the sample cuvette blocked so that it could not illuminate the detector. Figure 16 shows a schematic of the sample geometry with respect to the excitating laser and the output emission. The sample used in this case was rhodamine-575 in methanol at $460 \mu \mathrm{M}$. At this concentration $99 \%$ of the excitation was absorbed in the first $0.05 \mathrm{~cm}$ of the sample. We were not interested in measuring the emission resulting directly from this excitation but in the emission that followed the selfabsorption of this first emission. The mask shown in Fig. 16 achieved this condition. The transient emission spectrum resulting from this configuration is the upper plot in Fig. 17. When the mask was removed, we obtained the lower plot shown in Fig. 17. The solid curves superimposed on these two spectra will be discussed in the theoretical section. It was evident that there were significant distortions of the single exponential decay when the initially excited portion of the sample was masked. 


\section{G. Dye Degradation Measurements: Preliminary Results}

We have previously reported results for apparent degradation rates of rhodamine -575 and coumarin -540 in PMMA under accelerated and simulated solar exposure in LSC-1. We were interested in observing rates of degradation under actual solar exposure. Subsequently we have performed several experiments to pursue the phenomenon further.

The first sample tested was a $76-\mu \mathrm{m}$ rhodamine-590 PMMA plate $3 \mathrm{~mm}$ thick. Acetic acid was added to the monomeric solution prior to polymerization to increase the solubility of the dye. A 2-week continuous exposure (336 total h) caused a decrease in the peak optical density from 3.98 to 2.79 , as measured on a Cary-14 spectrophotometer. From the change in peak o.d. we estimate that the bleaching rate is $\sim 10^{-6}$ molecules/ photon. This is typical of photodegradation rates measured under different circumstances for similar dyes. $^{6}$

Multiple dye plates were manufactured to our specifications. A two-dye plate composed of $140-\mu \mathrm{M}$ coumarin-540 and 97- $\mu \mathrm{M}$ rhodamine-590 in PMMA was exposed for $1 \mathrm{yr}$ (we estimate $\sim 1000 \mathrm{~h}$ of direct sunlight) to the Michigan sun filtered by a plate glass window. We measured the emission of this exposed plate by illuminating the sample with a dispersed tungsten lamp source. The spectral distribution of the tungsten lamp closely approximated that of a $3000 \mathrm{~K}$ blackbody. A silicon cell was brought into optical contact with the edge using ethylene glycol as an index matching fluid. The output short circuit current on the cell was measured directly with an HP-3466A digital multimeter. The emission of the plate exposed behind plate glass had dropped $\sim 30 \%$ compared with a similar sample that had been stored in the dark. The peak optical density of the rhodamine peak had also been reduced from 2.7 to 2.2 during exposure or a drop of $20 \%$, while the coumarin peak had been reduced from 2.3 to 1.7 or a drop of $\sim 30 \%$. A section of the same unexposed plate was exposed to direct sunlight for 243 total h, with a resulting drop in peak emission of $\sim 30 \%$ and of the peak o.d. of the coumarin peak of $\sim 20 \%$. It should be emphasized that these are results from a particular LSC sample.

A PMMA plate containing $210-\mu \mathrm{m}$ coumarin -540 , 81- $\mu \mathrm{M}$ rhodamine-590, and $22-\mu \mathrm{M}$ sulforhodamine- 640 was also exposed to direct insolation for 243 continuous h. The total emission from the plate decreased $50 \%$, as measured using the above technique. The peak o.d. of the three dyes decreased 52,50 , and $51 \%$, respectively. Additional samples of this plate material were exposed under the same conditions. However, they were protected by either a Schott GG400 UV cutoff filter or by an $20-\mathrm{cm}$ (8-in.) square soda-lime glass cover. Both of these protected samples were also sealed against the outside atmosphere with polyvinylchloride electrical tape. Figure 18 shows a plot of the absorption spectra of the three samples described plus an unexposed control. While the soda-lime glass and the $4000-\AA$ cutoff filter absorb more than $90 \%$ of the UV radiation content

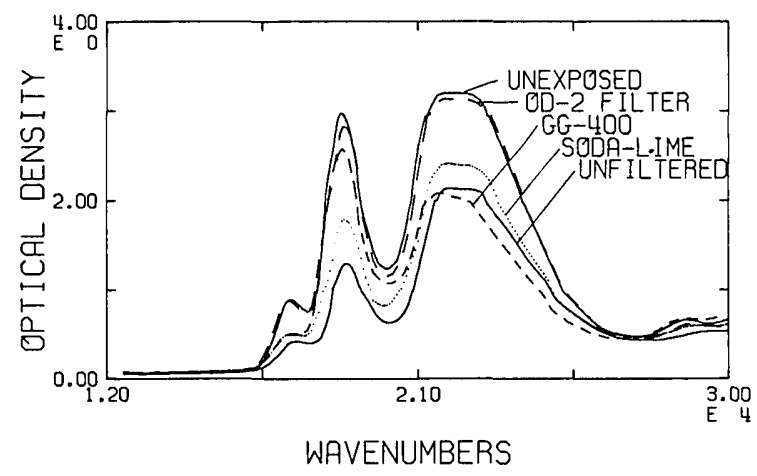

Fig. 18. Solar bleaching of a multiple-dye LSC plate. These are the absorption spectra for a PMMA plate containing $210-, 81-$, and $22-\mu \mathrm{M}$ concentrations of coumarin-540, rhodamine-590, and sulforhodamine-640, respectively. Four spectra correspond to 243 continuous $h$ of exposure through a 4000- $\AA$ cutoff filter, a soda-lime glass plate with no filter, and a control which was unexposed.

in sunlight, they had only a moderate effect at slowing the rate of photodegradation.

We made single-dye plates containing coumarin- 460 , 500 , and 540 , rhodamine- 590 and 610 , and oxazine- 725 in PMMA. After $\sim 250 \mathrm{~h}$ of continuous exposure, the emission had dropped $13 \%$ for coumarin-540, which was the best case, and the emission had dropped $66 \%$ for oxazine-725, which was the worst case, with the other dyes falling between these two limits. If the emission is assumed to decrease in a roughly exponential manner, these correspond to half-lives ranging from 300 to 3000 h (12-120 days).

Methanol solutions of the dyes coumarin-500, 535, and 540, rhodamine-590 and 640, sulforhodamine-640, cresyl violet-670, LD-700, and oxazine-720 were prepared in soda-lime glass vials $2.5 \mathrm{~cm}$ in diameter. Oxygen was removed from the samples by bubbling nitrogen gas through the solution for $1 \mathrm{~h}$ in a nitrogen atmosphere. The screw-on caps were sealed with RTV silicon rubber sealant. The optical density of the samples was measured every few days without disturbing the seals. Figure 19 shows the measured peak optical densities of these solutions as a function of total exposure time, which included hours of darkness. The measured optical densities typically did not decay in an exponential manner, but instead they decayed at rates that usually accelerated with time. The maximum exposure time was $432 \mathrm{~h}$ (18 days). This was insufficient time to obtain accurate decay rates for the longer lifetime dyes. Using an exponential as a rough approximation to the time-dependent optical density, the lifetime for the solutions ranged from $50 \mathrm{~h}$ for rhodamine-640 to $\sim 10,000 \mathrm{~h}$ (1 $\mathrm{yr}$ ) for rhodamine-590. Among the relatively stable dyes were coumarin -540 $(3000 \mathrm{~h})$, oxazine-720 (2000 h), and cresyl violet (7000 h).

Finally we measured photodegradation rates for five of the most stable dyes in a degassed environment. Methanol solutions of coumarin-540, rhodamine-590, sulforhodamine- 640 , cresyl violet- 670 , and oxazine- 750 


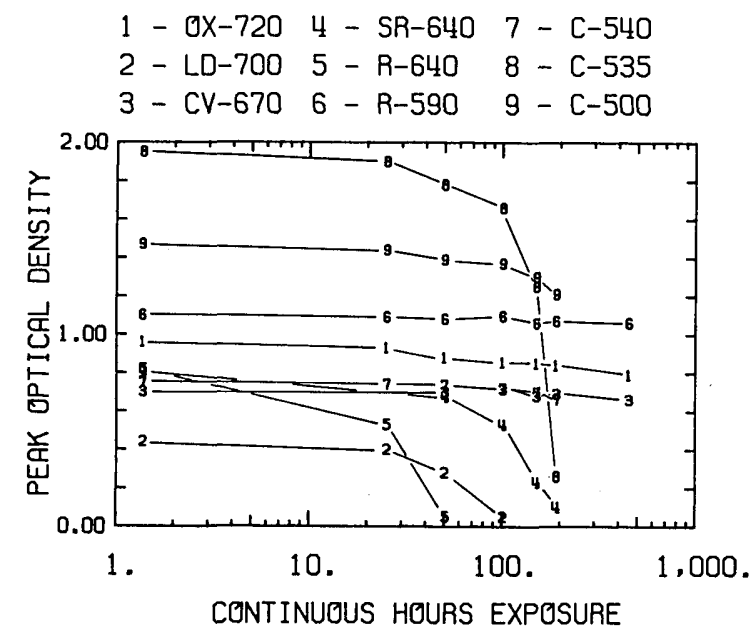

Fig. 19. Peak optical densities of methanol dye solutions under continuous solar exposure: 2.5 -cm i.d. soda-lime glass bottles were filled with solutions of oxazine-720 perchlorate, LD-700 perchlorate, cresyl violet-670 perchlorate, rhodamine-590 perchlorate, coumarin-540, coumarin-535, or coumarin-500. Screw-on caps were sealed with RTV. Absorption spectra were taken without disturbing the seals.

Table II. Performance Parameters of LSCs

\begin{tabular}{lclcccc}
\hline Device & No. Dyes & Matrix & $\begin{array}{c}\text { Geometric } \\
\text { Gain }\end{array}$ & $\begin{array}{c}\text { Flux } \\
\text { Gain }\end{array}$ & $\begin{array}{c}\text { Assumed } \\
\text { Cell } \\
\text { Eff. }\end{array}$ & $\begin{array}{c}\text { Collector } \\
\text { Eff. }\end{array}$ \\
\hline$A^{*}$ & 2 & PMMA & 23. & 2.1 & $18 \%$ & $1.9 \%$ \\
$B^{\dagger}$ & & thin film & 11. & 1.3 & $21 \%$ & $2.5 \%$ \\
$C^{*}$ & 3 & Glycol & 36. & 3.8 & $18 \%$ & $1.9 \%$ \\
$D^{\dagger}$ & 3 & thin film & 11. & 1.7 & $21 \%$ & $3.2 \%$ \\
E $^{*}$ & 1 & PMMA & 68. & 5.1 & $18 \%$ & $1.3 \%$ \\
& & & & & &
\end{tabular}

*Present work.

${ }^{\dagger}$ P. S. Friedman, LSC Contract Report, Owen-IHinois, SERI Contract XS-9-8216:1, 1980.

were placed in quartz cuvettes with outer dimensions of $1.3 \times 1.3 \times 6 \mathrm{~cm}$. The concentrations of the solutions were adjusted so that the peak optical density across the cuvette was between 0.5 and 1 . These samples were degassed by six freeze-pump-thaw cycles on a vacuum manifold and then flame sealed. The resulting lifetimes were $3.3,10 ., 36 ., 0.3$, and 1.0 direct sunlight $h$, respectively. The lifetimes could have been affected by the high UV transmission of the quartz cuvettes.

\section{H. Prototype Efficiency Measurements}

A variety of LSC devices have been tested by ourselves and others. Table II is intended to be a representative list of typical performance parameters. The geometric gain is again the ratio of the area exposed to the sun to the active area of the edge. The flux gain is the factor by which the short circuit current increases when attached to the plate, as opposed to facing the sun directly. The cell efficiency is the measured or assumed AM1 efficiency of the solar cells used (which in all cases were silicon). The collector efficiency is the total electrical power out divided by the total sunlight power incident on the plate.

In Table II devices $B$ and $D$ were built and tested by Owens-Illinois. The dyes were contained in thin plastic films attached to the surface of a clear substrate. Measurements were made under actual insolation, with the plate edges roughened and blacked where cells were not mounted. These plates have achieved the highest efficiencies, but their small geometric gains make them somewhat ineffective as concentrators. For example, cells mounted on LSC device $D$ will have only a $70 \%$ increase in output over an equal area of cells facing the sun directly.

Device $C$ was constructed by glueing two $110 \times 110$ $\times 0.3-\mathrm{cm}$ Plexiglas plates on opposing sides of $0.08-\mathrm{cm}$ Plexiglas spacers. The resulting gap between the plates was filled with a glycol solution containing $5.7 \times 10^{-5}$, $9.4 \times 10^{-5}$, and $5.1 \times 10^{-5} \mathrm{M}$ of coumarin-540, rhodamine-590, and rhodamine-640, respectively. The edges of the sandwich assembly were blacked with electrician's tape except for the portion coupled to the cell. The flux gain was measured under actual insolation by first measuring the short circuit current on a standard cell under direct insolation and then coupling the same cell to the plate, using a glycol film for optical matching and measuring the new short circuit current. We calculate that this dye combination will absorb $\sim 30 \%$ of an AMO spectrum in a two-pass geometry. The collector efficiency given in Table II is the cell AM1 efficiency times the flux gain divided by the geometric gain. This assumes a similar efficiency of the solar cell with and without the LSC (see LSC-1).

Device $E$ was a $120-\times 100-\times 0.4-\mathrm{cm}$ PMMA plate containing $2.2-\times 10^{-4}-\mathrm{M} \mathrm{DCM}$ (see later sections). The edges were taped as before except where the cells were mounted. The measurements were made using two cells with AM1 efficiencies matched to within $5 \%$ and both with an active area of $0.4 \times 2 \mathrm{~cm}$. One cell was mounted coplanar to the LSC surface as a reference, the other was contacted with the LSC edge using glycol as an index-matching fluid. The tests were made using a xenon flashlamp AMO simulator at JPL. The flux gain was determined to be the ratio of the peak power of the LSC-mounted cell and the reference cell, corrected for flash-to-flash variations. We calculate that this plate should absorb $30 \%$ of an incident AMO spectrum. The collector efficiency was determined in the same manner as for device $C$.

DCM and its possible variations appear to be a promising LSC dye due to its broad bands and large Stokes shift. The absorption, emission, and excitation spectrum for DCM cast in PMMA is shown in Fig. 20. Arrows $A$ and $E$ indicate the absorption and emission peaks in methanol, respectively. We observed that the Stokes shift is considerably diminished in PMMA. [The critical optical density (CODE) for DCM in PMMA is about 20.] We are presently investigating the suggestion of Sah et al. ${ }^{7}$ that DMSO added to the monomer prior to polymerization restores much of the solution Stokes shift of similar dyes. 


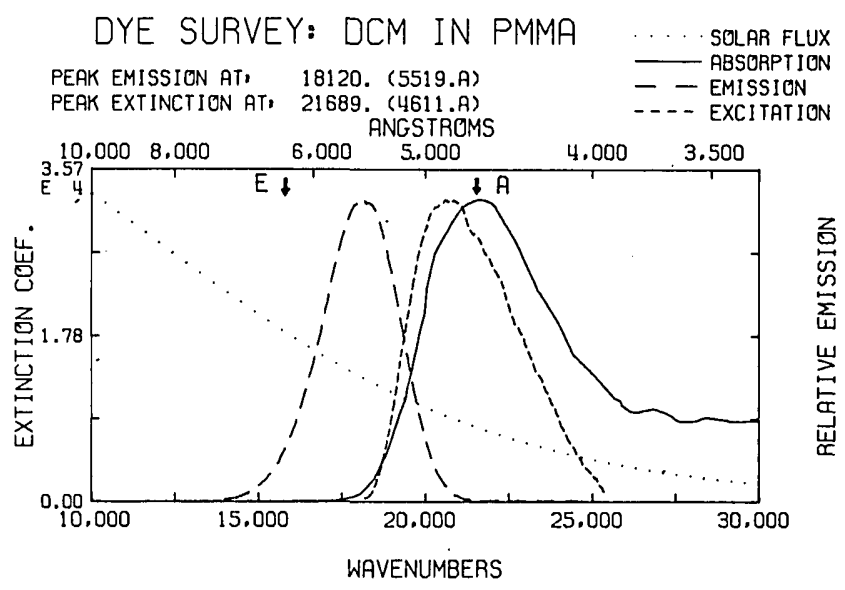

Fig. 20. Absorption, emission, and excitation spectra for DCM in PMMA. Arrows $A$ and $E$ indicate the absorption and emission maxima, respectively, for DCM in methanol. Adding DMSO to the monomer prior to polarimerization should substantially increase the Stokes shift in PMMA. ${ }^{7}$

\section{Theory}

We will first provide the principal relations derived in LSC-1 for predicting the performance of an LSC. To determine a figure or merit for LSC performance, we will develop similar relations for a purely scattering plate. Finally we will restate our self-absorption model for the large anti-Stokes shifted limit, and we will derive relations for calculating self-absorption rates from measured steady-state spectroscopic and polarization data and from transient emission measurements.

\section{A. Preliminaries}

By way of review, we note that if $A_{f}$ is the total area of the face exposed to direct sunlight and $A_{e}$ is the area of the edge contacting the solar cells, the geometric gain of the concentrator is $G_{\text {geom }}=A_{\text {face }} / A_{\text {edge. }}$. If $n$ is the index of refraction of the LSC plate material, by Snell's law we find that the probability that isotropic luminescence (for the more general case see Ref. 12) will escape out of the surfaces of the plate rather than being trapped by total internal reflection is $P=1-(1-1)$ $\left.n^{2}\right)^{1 / 2}$. Light which escapes from the LSC plate because it was incident to the surface at less than the critical angle of incidence is said to escape through the critical escape cones. The fraction of the solar flux initially absorbed by the plate which is transmitted to the solar cells is Q [LSC-1, Eq. (23)]:

$$
Q=\frac{(1-r)(1-P) \eta}{1-\eta[r P+(1-P) \cdot r]},
$$

where $r$ is the average probability that a luminescent photon will be self-absorbed while traversing the LSC plate, $\bar{r}$ is the probability that a similar self-absorption will occur within the critical escape cone, and $\eta$ is the quantum efficiency of luminescence for the given luminescing center. The approximations made in Eq. (1) are that trapped luminescence is not scattered by bulk or surface imperfections, that the level of excitation of the plate is well below the coherence limit, and that only predominantly homogeneously broadened luminescent species are used in the plate. The result applies to a cascade of luminescing centers in the limit that the efficiency of energy transfer to the final or lowest energy center is $100 \%$.

Before treating the real case, it is instructive to calculate the collection efficiency of a purely scattering plate. Consider the planar solar concentrator (PSC) geometry of LSC made from a ribbon of clear material having a thickness $T$ and width $W$. We hypothesize that there is a plane of isotropic scattering centers located a distance $T / 2$ from either exposed surface, so that light impinging upon this scattering plane is isotropically scattered, with a scattering efficiency of $\eta_{\text {scat }}$. Cells are mounted along both edges. The probability that scattered light from a point on the scattering plane a distance $L$ from one of the edge mounted array of cells will be incident on that array of cells is $(1 / \pi) \tan ^{-1}(T / L)$ (see Appendix A). Ignoring multiply scattered light, the fraction of uniform scattering by the entire scattering plane that is collected by both edge-mounted cell arrays is

$$
F=\frac{2 T}{W \pi} \cdot\left\{\frac{W}{T} \tan ^{-1}(T / W)=1 / 2 \ln \left[1+(W / T)^{2}\right]\right\} .
$$

So if light is incident on this scattering plate, we find that the initial fraction of that flux which is collected and transferred directly to the cells without further scattering, called the first generation collection probability, is

$$
Q^{(1)}=\eta_{\text {scat }} \cdot F .
$$

Light will return to the scattering plane if it is not collected by the cells and if it does not escape out to the face. If $(1-F)(1-P)$ is the probability that scattered light is trapped by total internal reflection and is not collected by the edge-mounted cells, we find that the second generation collection probability is

$$
Q^{(2)}=\eta_{\text {scat }}^{2} \cdot F(1-F)(1-P) \text {. }
$$

In a similar manner, we find that the $i$ th generation collection probability is

$$
Q^{(i)}=\eta_{\text {scat }} \cdot F\left[\eta_{\text {scat }}(1-F)(1-P)\right]^{i-1} .
$$

The total collection probability is the sum of the individual probabilities for each generation:

$$
Q=\frac{\eta_{\text {scat }} \cdot F}{1-(1-F)(1-P) \eta_{\text {scat }}} .
$$

The average number of times that collected light has been scattered from the scattering plane is the sum of the collection probabilities times their generation number:

$$
N_{e}=\sum_{i=1}^{\infty} i Q^{(i)} / Q .
$$

If we make the approximations that except for the scattering plane the matrix is perfectly transparent, that the AM1 efficiency of these photovoltaic cells $\eta_{\mathrm{pvc}}$ is independent of the light intensity, and that the scattering plane scatters all the incident sunlight, the total collector efficiency $\eta_{c}$ (electrical power out per solar power in) is 


$$
\eta_{c}=\frac{\eta_{\mathrm{pvc}} \eta_{\mathrm{scat}} F}{1-(1-F)(1-P) \eta_{\mathrm{scat}}} .
$$

The potential broadband spectral characteristics and insensitivity of scattering centers to degradation suggest that a scattering plate might have interesting applications for systems requiring only modest flux gains.

\section{B. Steady-State Self-Absorption}

In LSC-1 we derived a recursion relation for computing the intensity and spectrum of self-absorbed emission in the limit that the anti-Stokes shift is small. We will begin by presenting a similar formalism for the limiting case of unrestricted anti-Stokes shifting of the emission with respect to the energy of the absorbed excitation, i.e., the emission spectrum of the luminescing species is independent of the excitation energy. For clarity, we will restrict ourselves to the case of a semiinfinite LSC rod. Next we will provide a simplified form for calculating the self-absorption probability from known absorption and emission spectra. This probability can also be deduced experimentally from the anisotropy of the polarization of the emission from a polarized excitation or from the change in lifetime as a function of the concentration and path length through which the emission travels.

Consider a semi-infinite rod of LSC material having a diameter $d$ and containing luminescing centers at a concentration of $C$ moles per liter, with an extinction coefficient $\epsilon(\bar{\nu})$ and a normalized luminescence spectrum $f(\bar{\nu})$. $S C$ is the scattering coefficient corresponding to losses in the rod other than self-absorption (e.g., matrix absorption and scattering). $P$ is the probability that luminescence will escape out of the side of the rod, which has the form $P=1 / n$ in the case of a cylindrical rod, where $n$ is the index of refraction of the rod material. We define $\Lambda(x, y)$ to be a function specifying the probability that an emission originating at a position $x$ will be absorbed at a position y (see Appendix $\mathrm{C}$ ):

$$
\Lambda(x, y)=\frac{\ln (10) \cdot C}{2} \int_{0}^{\infty} d \bar{v} f(\bar{\nu}) \epsilon(\bar{\nu}) \int_{A}^{B} d z \frac{\exp (-z)}{z},
$$

where

$$
\begin{aligned}
& z=|x-y| \cdot[\ln 10 \cdot C \cdot \epsilon(\bar{\nu})+S C], \\
& A=[\ln (10) \cdot C \cdot \epsilon(\bar{\nu})+S C] \cdot|x-y|, \\
& B=\left\{\begin{array}{l}
{[\ln (10) \cdot C \cdot \epsilon(\bar{\nu})+S C] \cdot|x-y| / \sin \theta_{c}|x-y|>\frac{d}{2} \tan \theta_{c},} \\
{[\ln (10) \cdot C \cdot \epsilon(\bar{\nu})+S C] \sqrt{\frac{d^{2}}{4}+(x-y)^{2}}|x-y|<\frac{d}{2} \tan \theta_{c} .}
\end{array}\right.
\end{aligned}
$$

Equation (6) assumes that absorbed excitation anywhere in an infinitesimal disk at $x$ is emitted from a point at the center of the disk. Next we define $\Xi_{(x)}^{(i)}$ to be the spatial distribution of the $i$ th generation of excitations along the rod. For example, if the rod is initially excited by a focused light source at a position $x$, $\Xi^{(1)}$ is a delta function at position $x$ :

$$
\Xi^{(1)}(x)=I_{0} \delta(x) \text {. }
$$

Excitations directly due to absorption of the externally incident light are first generation excitations. Second

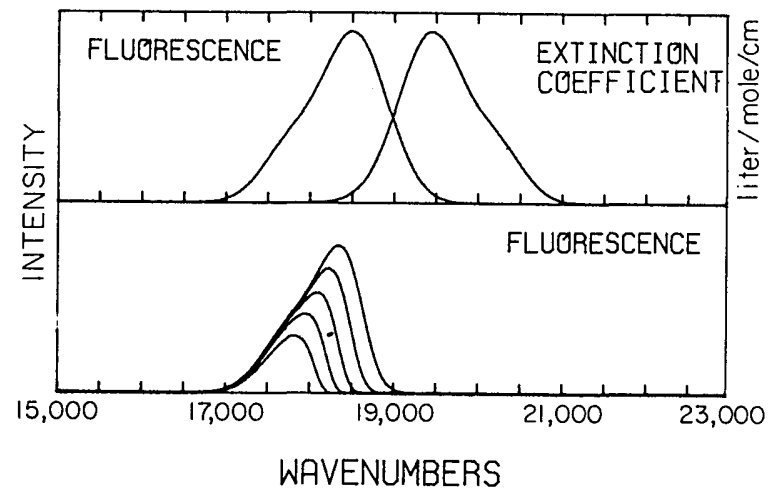

Fig. 21. Calculated spectral and intensity changes due to self-absorption. Experimental absorption and emission spectra of rhodamine- 575 were approximated by the sum of two Gaussians. Stokes shift of 1000 wave numbers reproduces the typical overlap between these two spectra. Using Eqs. (6)-(8), we have analytically imitated the experiment shown in Fig. 11 using the same geometry, concentration, and path lengths.

generation excitations arise from the self-absorption of first generation emissions. For example,

$$
\Xi^{(2)}(x)=\eta \int_{0}^{\infty} d y \Xi^{(1)}(y) \Lambda(x, y),
$$

and in general

$$
\Xi^{(i)}(x)=\eta \int_{0}^{\infty} d y \Xi^{(i-1)}(y) \Lambda(x, y),
$$

where $\eta$ is the quantum efficiency of luminescence. The luminescence spectrum observed at the end of the rod $(x=0)$ is the sum of the intensities due to all the different generations of excitations in each element:

$$
\begin{aligned}
Q(\bar{\nu})= & \eta \int_{0}^{\infty} d x \int_{0}^{\pi / 2-\theta_{c}} \\
& \times \sin \theta d \theta \exp \{-x[\ln (10) \cdot C \cdot \epsilon(\bar{\nu})+S C] / \cos \theta\} \\
& \times f(\bar{\nu}) \cdot \sum_{i=1}^{\infty} \Xi^{(i)}(x)
\end{aligned}
$$

In the limiting case that the Stokes shift is large, the probability for reabsorbing the emission is approximately zero everywhere or $\Lambda(x, y)=0$. Therefore, $\Xi^{(i)}(x)=0$ for all $i>1$, by Eq. (7c), and only the first generation emission is collected.

Figure 21 shows the results for $Q(\bar{\nu})$ vs $\bar{\nu}$ for two numerically generated absorption and emission $[\epsilon(\bar{\nu})$ and $f(\bar{\nu})$ of Eq. (6)] spectra similar in form to the absorption and fluorescence spectra of rhodamine-575. The path lengths, rod diameters, and dye concentrations are the same as for the experimental data shown in Fig. 11. The scattering coefficient used in this calculation is $S C$ $=0.008 \mathrm{~cm}^{-1}$. The absorption spectrum is the sum of two Gaussians with peak positions of 19,500 and 20,300 $\mathrm{cm}^{-1}$, peak widths of 840 and $680 \mathrm{~cm}^{-1}$, and peak heights of 94,000 and $27,000 \mathrm{liters} / \mathrm{mole} / \mathrm{cm}$, respectively. The emission spectrum was the same sum of two Gaussians reflected in a mirror line at $19,000 \mathrm{~cm}^{-1}$ and - normalized to unity. Notice the shift in spectral position with increasing self-absorption is accompanied by 


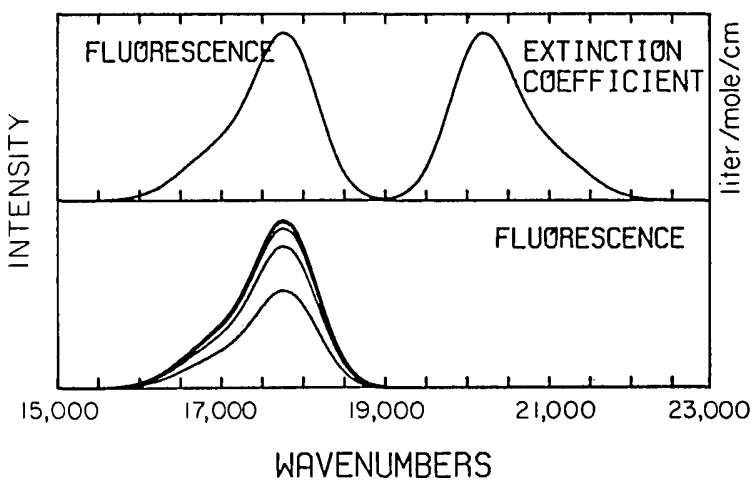

Fig. 22. Calculated spectral and intensity changes due to self-absorption. Same calculation was made as in Fig. 21, except that the Stokes shift was increased to 2500 wave numbers.

a decrease in collected intensity. Figure 22 shows the results of a similar calculation in which the Stokes shift between the absorption and emission spectrum has been increased from 1000 to $2500 \mathrm{~cm}^{-1}$ to lower their overlap. While the nonzero scattering losses cause an overall decrease in the intensity with increasing path length, the emission spectrum is seen not to shift appreciably. [Note: In LSC-I an additional $(1 / \cos \theta)$ factor is required in the finite element analysis of Appendix II in order that each element have the same initial intensity with respect to a point on the edge.]

\section{Self-Absorption Probability}

While the preceding technique is useful in obtaining spectral information about the emission from an LSC rod, a simpler calculation appears to be a reasonably good approximation for calculating self-absorption probabilities. In the simplest 1-D case, the probability that self-absorption will occur is found by the BeersLambert law:

$$
r=\int_{0}^{\infty} d \bar{v} f(\bar{\nu})\left[1-10^{-x} C_{\epsilon}(\bar{\nu})\right],
$$

$r$ is the probability that an emission, having the normalized distribution $f(\bar{\nu})$, will be self-absorbed by the luminescing centers having an extinction coefficient $\epsilon(\bar{\nu})$ and a concentration $C$ over a path length $x$. The approximation that we will make in this section is that the probability of self-absorption for emission in an LSC plate is given by Eq. (9), where $C$ is the concentration of dye in the plate, and $x$ is the characteristic length ${ }^{8}$ of the LSC plate. The characteristic length of a square LSC is the length of the side of the square. The characteristic length for self-absorption in the critical cone is the thickness of the LSC plate containing the luminescing centers.

Clearly this is a gross simplification of the proper averaging of the self-absorption probability over all path lengths and energies of possible emission. We justify this approximation by showing that the self-absorption probability has roughly a logarithmic dependence on path length (see Fig. 25, for example). However, it is useful to explicitly calculate the weighted average path length traveled by light collected in an LSC and com-

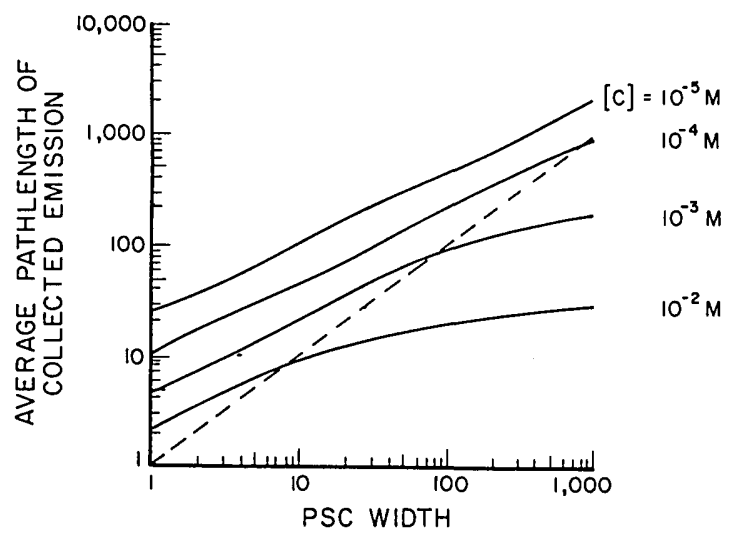

Fig. 23. Average path length of collected emission vs width of a PSC plate. Using the full PSC collection efficiency calculation from LSC-1, we have calculated the average distance through the plate traversed by emission which is collected at the edge of the plate for four different concentrations of rhodamine-575. Cells were assumed to be mounted on both edges of the ribbon. Plate thickness was assumed to be $1 \mathrm{~cm}$ so that the $10^{-5}-\mathrm{M}$ concentration corresponds to a peak o.d. of 1 . Dashed line indicates the characteristic length approximation that the average path length is equal to the plate width.

pare this result with that predicted by the characteristic length approximation.

We used the collection efficiency formalism of LSC-1 [Eq. (20)] to compute the first generation collection efficiency $Q^{(1)}$ for a PSC geometry LSC. We assumed that the width was variable, the thickness was $1 \mathrm{~cm}$, the index of refraction was 1.5, and cells were mounted on both edges of the ribbon. Since $Q^{(1)}=\eta(1-r)(1-P)$, we found the self-absorption probability $r$. Using Eq. (9), we found the average path length of the collected light in the plate that would result in the computed $r$. Figure 23 shows the results of this calculation, where we have calculated the average path length as a function of the plate width for four different concentrations of rhodamine-575. The dashed line indicates the characteristic length approximation, where the average path lengths are assumed to be the width of the plate. At low concentrations and PSC widths, the average path length is greater than the width, while at high concentrations and PSC widths, the average is less than the width.

\section{Polarization Anisotropy: a Second Self- Absorption Measurement}

Equation (1) tells us that the collection efficiency of the plate can be easily found once four parameters are known: $P$, the probability of escape out of the critical cones; $r$, the probability of self-absorption for trapped emission; $\bar{r}$, the probability of self-absorption within the critical cones; and $\eta$, the quantum efficiency of luminescence. $P$ is known from the index of refraction of the host material, while the quantum efficiency can be measured using standard techniques. ${ }^{9}$ Equation (9) gives a reasonably simple approximate result for numerically calculating the self-absorption probabilities if the average path length of travel of the emitted light in the plate is known. As we just showed, an exact 
calculation of the self-absorption probabilities is tractable for a few simple LSC geometries. In general, however, a simpler or more direct measurement of $r$ and $\bar{r}$ is desirable. Two techniques providing this measurement are emission polarization anisotropy and total lifetime lengthening.

Suppose we excite an LSC with vertically polarized light and detect either the vertically or horizontally polarized emission intensities emitted in a horizontal direction, as shown in Fig. 12. If the orientation of the absorption dipole moments of the luminescing centers are initially isotropic, the probability that any center will absorb an excitation as a function of its absorption dipole orientation is proportional to $[\cos (\theta)]^{2}$. Using a more concise notation, ${ }^{4,10}$ if $\hat{\epsilon}_{i}$ is a unit vector in the direction of the electric field of the polarized excitation, and $\bar{\mu}_{a}$ is the absorption dipole moment, the angular dependence of the excitation probability is given by $\left[\hat{\epsilon}_{i} \cdot \bar{\mu}_{a}\right]^{2}$. If we assume that the emission dipole moment $\bar{\mu}_{e}$ is parallel with the absorption dipole and that the orientation of these moments is fixed on the time scale of the total lifetime, the intensity of emitted light polarized in the direction $\hat{\epsilon}_{f}$ is given by (apart from a constant)

$$
I=\left\langle\left[\hat{\epsilon}_{i} \cdot \bar{\mu}_{a}\right]^{2}\left[\hat{\epsilon}_{f} \cdot \bar{\mu}_{e}\right]^{2}\right\rangle .
$$

The brackets \langle\rangle denote an average over the unit sphere. The emission intensity polarized parallel to the excitation is therefore given by

$$
\begin{aligned}
I_{\perp} & =\left\langle\left[\hat{\epsilon}_{i} \cdot \bar{\mu}_{a}\right]^{2}\left[\hat{\epsilon}_{i} \cdot \bar{\mu}_{e}\right]^{2}\right\rangle \\
& =\frac{\mu_{a}^{2} \mu_{e}^{2}}{4 \pi} \int_{0}^{2 \pi} d \phi \int_{0}^{\pi} d \theta \sin \theta \cos ^{4} \theta \\
& =\mu_{a}^{2} \mu_{e}^{2} / 5 .
\end{aligned}
$$

Similarly the emission intensity polarized in a direction perpendicular to the excitation is

$$
\begin{aligned}
I_{\|} & =1 / 2\left\langle\left[\hat{\epsilon}_{i} \cdot \bar{\mu}_{a}\right]^{2}\left[\hat{\epsilon}_{i} \times \bar{\mu}_{e}\right]^{2}\right\rangle \\
& =\frac{\mu_{a}^{2} \mu_{e}^{2}}{8 \pi} \int_{0}^{2 \pi} d \phi \int_{0}^{\pi} d \theta \sin ^{3} \theta \cos ^{2} \theta \\
& =\frac{\mu_{a}^{2} \mu_{e}^{2}}{15} .
\end{aligned}
$$

The reduced anisotropy is defined to be

$$
R A=\frac{I_{\|}-I_{\perp}}{I_{\|}+2 I_{\perp}} .
$$

The initial excitation has no perpendicular component, so its reduced anisotropy is 1 . From the intensities calculated in Eqs. (10c) and (10d), we see that the parallel polarized emission has three times the intensity of the perpendicular component giving $R A=0.4$ for the first generation emission. If the orientation of the luminescing dipole is different from that of the absorption dipole, the reduced anisotropy decreases by a factor $e$ (Ref. 4):

$$
R A=2 e / 5 \quad 0 \leqslant e \leqslant 1 .
$$

Emission from an LSC due to polarized excitation displaying a reduced anisotropy of $2 e / 5$ can therefore be identified as emission from the first generation of excitations. The reduced anisotropy due to the self- absorption of this emission and its subsequent reemission are given by (see Appendix B)

$$
R A^{(2)}=(2 / 5)^{3}
$$

in the limit of parallel moments fixed in space. In general, the reduced anisotropy of the $i$ th generation's emission is

$$
R A^{(i)}=(2 / 5)^{(2 i-1)} .
$$

Suppose we measure the reduced anisotropy of the emission from an LSC due to a polarized excitation. From Eq. $(10 \mathrm{~g})$, we know that the measured $R A$ is the sum of $R A^{(i)}$ for each generation times the collection probability for that generation; assuming $Q_{2}$ is polarization independent,

$$
R A_{\exp }=\sum_{i=1}^{\infty} R A^{(i)} Q^{(i)} / Q .
$$

In the limit of very low concentration luminescing centers, there is no self-absorption, so $Q^{(1)}=Q, Q^{(i)}(i$ $>1)=0$, and $R A_{\exp }=2 e / 5$. For higher concentrations we use the technique of averaging over each generation, as in LSC-1, Eq. (23). If we assume by analogy that the $i$ th generation has $R A^{(i)}=(2 e / 5)^{2 i-1}$,

$$
\begin{aligned}
R A_{\exp }= & {\left[\frac{2 e}{5} \eta(1-r)(1-P)+\left(\frac{2 e}{5}\right)^{3}\right.} \\
& \times \eta^{2}(1-r)(1-P)[\bar{r} P+r(1-P)] \\
& \left.+\left(\frac{2 e}{5}\right)^{5} \eta^{3}(1-r)(1-P)[\bar{r} P+r(1-P)]^{2}+\cdots\right] / Q \\
& =\frac{2 e}{5} \cdot \frac{1-\eta(\bar{r} P+r(1-P))}{1-\eta\left(\frac{2 e}{5}\right)^{2}[\bar{r} P+r(1-P)]} .
\end{aligned}
$$

If the excitation is near enough to the edge of the plate so that the probability of self-absorption inside and outside of the critical cones is about the same, $r=\bar{r}$, then

$$
R A_{\text {exp }}=\frac{2 e}{5} \frac{1-n r}{1-\left(\frac{2 e}{5}\right)^{2} \eta r} \simeq \frac{2 e}{5}(1-\eta r) .
$$

Similar simplifications arise if the measurement is arranged so that $P$ is 0 .

\section{E. Lifetime Lengthening: a Third Self-Absorption Measurement}

The rate of self-absorption both inside and outside of the critical cones can be calculated directly from measured transient emission spectra. At $t=0$, suppose that there are $n_{0}$ excitations in a dye ensemble. The rate of change of this population is proportional to the population:

$$
\frac{\partial}{\partial t} n^{(1)}(t)=-\frac{n^{(1)}(t)}{\tau} \quad n^{(1)}(0)=n_{0}
$$

where $\tau$ is the total lifetime. We assume that there is no transit time dispersion due to emission from different parts of the sample or that the lifetime is long compared with the transit time for the light in the sample. Since typical fluorescent dyes have lifetimes of the order of nanoseconds, the applicability of this formalism is re- 
stricted to devices a foot across or smaller. If $r$ is the probability that the emission is self-absorbed if it is emitted outside of the critical cones, and $\bar{r}$ is the probability of self-absorption within the critical cones, $P$ is the probability of trapping in the critical cones, and $\eta$ is the quantum efficiency of luminescence, then the kinetic equation for the number of second generation excitations as a function of time $n^{(2)}(t)$, resulting from first generation emissions, is

$$
\frac{\partial}{\partial t} n^{(2)}(t)=-\frac{n^{(2)}(t)}{\tau}+\eta n^{(1)}(t) \cdot[\bar{r} P+r(1-P)] / \tau .
$$

Note that $\tau=\tau_{\text {rad }} \cdot \eta$, where $\tau_{\text {rad }}$ is the radiative lifetime.

We will assume that the probability of self-absorption is independent of the number of emissions previously experienced by the excitation. This is equivalent to assuming the limit of a predominantly homogeneously broadened ensemble at temperatures sufficiently high to allow anti-Stokes shift of the emission which is large compared with the emission linewidth. The third generation excitations therefore are

$$
\frac{\partial}{\partial t} n^{(3)}(t)=-n^{(3)}(t) / \tau+\eta n^{(2)}(t)[\bar{r} P+r(1-P)] / \tau .
$$

These form a system of equations with the solutions

$$
\begin{aligned}
& n^{(1)}(t)=n_{0} \exp (-t / \tau), \\
& n^{(2)}(t)=n_{0} \frac{t}{\tau} \eta[\vec{r} P+r(1-P)] \exp (-t / \tau), \\
& n^{(i)}(t)=n_{0}\left\{\frac{t}{\tau} \eta[\vec{r} P+r(1-P)]\right\}^{i-1} \exp (-t / \tau) /(i-1) !
\end{aligned}
$$

Summing the various generations gives the number of excitations in the ensemble as a function of time:

$$
n(t)=\sum_{i=1}^{\infty} n^{(i)}(t)=n_{0} \exp \left(-\frac{t}{\tau}\{1-\eta[\bar{r} P+r(1-P)]\}\right) .
$$

Thus self-absorbed emission will have an apparent lifetime which is lengthened by a factor $1 /\{1-\eta[\bar{r} P+$ $r(1-P)]\}$. This factor is equal to one if $r=\bar{r}=0$. Measurements of the experimental transient emission decay from an excitation which is short compared with the total radiative lifetime at low concentrations can therefore give $\tau, \tau /(1-\mathrm{r} \eta)$, and $\tau /\{1-\eta[\bar{r} P+r(1-P)]\}$ for the three cases of an extremely dilute sample, edge emission due to an excitation near to the edge, and from general illumination of the plate, respectively.

If each generation of excitations is distributed throughout the sample in such a way that the average probability of emission from each generation reaching the detector is the same, the transient emission spectrum should have the simple exponential behavior of Eq. (14). It is possible, however, to configure the exciting source and sample in such a way that the first generation emission has a very different probability of reaching the detector. For example, if the exciting laser pulse is at an energy corresponding to the absorption maximum for the emitting center, nearly all the laser light will be absorbed in typically less than the first few millimeters of the sample. A mask can then be placed between this excited region and the detector, so that the excited region of the sample can illuminate the rest of the sample without illuminating the detector (Fig. 16). Assuming that none of the first generation emission illuminates the detector due to scatter in the rest of the sample and $r=\bar{r}$, we find that the measured transient spectrum should be proportional to the usual total decay of the population minus the missing first generation emission

$$
n_{m}(t)=n_{0} \cdot\{\exp [-t(1-r \eta) / \tau]-\exp (-t / \tau)\}=n(t)-n^{(1)}(t) .
$$

If the probability of self-absorption is high enough, a significant fraction of the first generation emission can be reabsorbed in the masked region. In this case, some fraction of the higher-order generations will have to be subtracted. Because of the sensitivity to scattering and inadvertent masking of higher-order generations, this technique is inferior to the simple lifetime measurements for determining self-absorption probabilities.

\section{F. Effects of Homogeneous Broadening}

The experimental results of the previous section encourage us to assume that the absorption and emission spectra are predominantly homogeneously broadened, at least for the case of organic dyes dissolved in solvents or diffused into plastics. More specifically, this approximation allows a complete calculation of the efficiency of a PSC or ribbon geometry device using the first generation collection efficiency [LSC-1, Eq. (20)].

Since the first generation collection efficiency is defined to be $\eta(1-r)(1-P)$, where $P$ is the critical cone escape probability and $r$ is the self-absorption probability outside of the critical cones, we can solve for the total collection efficiency from Eq. (1). Solving for $r$ in terms of $Q^{(1)}(\mathrm{PSC})$ and substituting in Eq. (1), we find

$$
Q_{\mathrm{PSC}}=Q_{\mathrm{PSC}}^{(1)} /\left\{1+Q_{\mathrm{PSC}}^{(1)}-\eta[1-P(1-\bar{r})]\right\},
$$

where $\bar{r}$ is the self-absorption probability inside the critical cones. Equation (16) gives a tractable calculation of the efficiency of the PSC device with only two further approximations. The first approximation is that the self-absorption probability in the critical cones can be computed using Eq. (9). The second is that we must assume that the self-absorbed emission is distributed isotropically across the plane of the PSC plate. The latter approximation should be expected to break down only for high concentrations of luminescing species and then only in the region near the solar cells. The reason one would expect the approximation to break down in this case is that if the concentration is high, self-absorption occurs in a path length which is short compared with the width of the plate, so that it is possible for one region of the plate to contain a different excitation density than another region. Since the solar cells do not emit light, the region near the cells is not illuminated from all sides, as are other portions of the plate. If the luminescing species are predominantly inhomogeneously broadened, appropriate averaging must be done over all homogeneous subensembles and over all $\mathrm{cm}^{-1}$.

In summary, we have suggested three ways to deter- 
mine self-absorption rates in an LSC: spectral convolution; steady-state emission polarization anisotropy; and transient lifetime lengthening. Additional techniques exist. For example, the peak of the emission spectrum in Fig. 11 moves toward lower energy as selfabsorption increases. This allows for an indirect measure of the self-absorption probability if these peak positions are calibrated using one of the above techniques.

\section{Discussion of Results}

\section{A. Spectral Homogeneity}

As mentioned before, the relative homogeneity of absorption and emission spectra of organic dyes is dependent on the host material. For example, in Fig. 4 we see that the maximum of the emission spectrum for rhodamine-575 in methanol at room temperature is independent of the excitation energy, even for excitations which are $2000 \mathrm{~cm}^{-1}$ lower in energy than the emission peak. This blue-shifted emission disappears when the sample is cooled to liquid nitrogen temperatures, so that this extra energy in the emission must come from thermal energy in the molecule or from the thermal bath. If the same dye is diffused into a PMMA plate, essentially the same results are produced, as shown in Fig. 6. We therefore concluded that, for these two host materials, the absorption and emission spectra are predominantly homogeneously broadened. Dyes which were dissolved in methyl methacrylate monomer and then polymerized displayed characteristics of inhomogeneous broadening. As shown in Fig. 5, the emission spectrum is dependent on the precise energy of the excitation; it was possible to select portions of the ensemble of dye molecules which displayed significantly different spectral characteristics than the ensemble average.

We have seen that dye molecules in a host material at room temperature are able to emit light of several thousand wave numbers greater energy than the initial excitation. This is not surprising, since these molecules typically have a large number of internal degrees of freedom. At liquid nitrogen temperatures, $k T$ is reduced by a factor of 4 , which is sufficient to decrease the available energy in the molecule so that these large blue-shifted emissions do not appear. This temperature sensitivity would imply that even larger blue-shifts of emission would be possible in plates at the elevated temperatures of roof-mounted collectors.

\section{B. Self-Absorption}

We can also infer that the cast PMMA samples showed stronger absorption in the extreme red tails than the solution or diffused samples. This can be seen by comparing the shape and the signal to noise for emission spectra in Figs. 4 and 5. This is very important for optimizing LSC efficiency, because it is the red absorption tail which leads to self-absorption losses.

A comparison of Figs. 11 and 21 shows that the numerical technique described in the theory section is capable of accurately modeling the attenuation and spectral shift of the emission due to self-absorption.
We observe that most of the emission spectrum overlaps sufficiently with the red absorption tail, so that most of the emission can be self-absorbed for concentrations and path lengths typical of LSC devices. The model also verifies that a large Stokes shift will eliminate self-absorption losses. This experiment also suggests that the probability of self-absorption might be measured simply by observing the peak position of the emission as a function of concentration or path length.

Clearly it is important to account for the self-absorption rates for luminescing species which are to be incorporated into an LSC. In general, the self-absorption rate is related in a complicated way to the geometry, the dye concentrations, and their absorption and emission spectra. For the purpose of designing devices, this interrelationship usually does not suggest, in a transparent way, what the proper species are and what their concentrations should be. We will therefore define a self-absorption parameter, the critical optical density, to be the peak optical density of a sample containing a luminescing species, so that the emission from the species has a $50 \%$ chance of being self-absorbed while traversing the sample. Mathematically, if a luminescing species has a normalized luminescence spectrum $f(\bar{\nu})$, a molar concentration $C$, and an extinction coefficient $\epsilon(\bar{\nu})$ with a maximum value of $\epsilon\left(\bar{\nu}_{m}\right)$, and this species is contained in a sample with an optical path length $L$, the critical optical density is

$$
\mathrm{CODE}=\epsilon\left(\bar{\nu}_{m}\right) C L,
$$

where the concentration and path length have been adjusted so that

$$
1 / 2=\int_{0}^{\infty} f(\bar{\nu}) d \bar{\nu} 10^{-C L \epsilon(\bar{\nu})}
$$

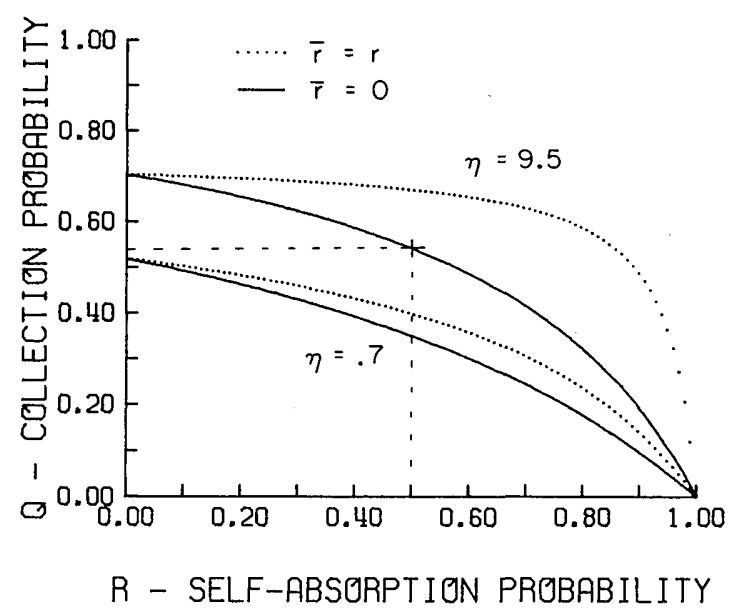

Fig. 24. Collection efficiency vs self-absorption probability. Assuming that $P=0.26$, we have used Eq. (1) to calculate the collection efficiencies for the cases where there is no self-absorption in the critical cones (solid lines) and where the self-absorption rates inside and outside of the critical cones are the same (dotted lines). Upper two curves assume a quantum efficiency of luminescence of 0.9 , while the lower two assume 0.7 . Vertical line at $r=0.5$ indicates the selfabsorption rate at the CODE. 
The CODE is the radiative analog to the Forster critical concentration. It is useful because it characterizes the efficiency of an LSC plate as a function of size or geometric gain. In Fig. 24, we have plotted the collection efficiency of an arbitrary LSC plate as a function of self-absorption probability using Eq. (1). The index of refraction is assumed to be 1.49 , the solid lines refer to the case where there is no self-absorption in the critical cones, and the dotted lines are for the case where self-absorption rates are equal inside and outside of the critical cones. The vertical line originating at $r=0.5$ intersects the curves at the CODE operating point. For example, a typical LSC plate will have an index of refraction of 1.5 or an escape probability of $P=0.26$, a quantum efficiency of luminescence of $\eta=0.9$, and negligible self-absorption in the critical cone. If the self-absorption probability is 0.5 , we find from Fig. 24 that the collection efficiency of the plate is also $\sim 0.5$. To obtain reasonably good absorption of sunlight or emission from other dyes, the peak optical density of the final dye must be at least 1 across the thickness of the LSC plate. In this case, the CODE will be the number of plate thicknesses which the emission can traverse before being $50 \%$ self-absorbed. Since the geometric gain of a plate is roughly the width of a plate divided by its thickness, the CODE is roughly the maximum geometric gain of a plate, using the particular luminescing center as a single or final dye in a cascade, which produces a collection efficiency of 0.5 .

Table I gives the critical optical densities for the organic laser dyes tested. The $30 \%$ errors are due to uncertainty in the measurement of the absorption tails. Typical values for the CODE are 20. Such dyes would produce collection efficiencies of $<0.5$ if incorporated in plates with a geometric gain greater than 20 . Several dyes have critical optical density around 80 , while DCM (4-dicyanomethylene-2-methyl-6-p-dimethylaminostyryl-4H-pyran) has a critical optical density of $\sim 250$.

We have shown results on three different measurements of the effects of self-absorption in rhodamine575: spectral overlap; emission depolarization; and lifetime broadening. The critical optical density for rhodamine-575, from a spectral overlap calculation using Eq. (16b), implies that self-absorption will have substantial effects on the performance of an LSC. We are therefore interested in the consistency of these different measurement techniques in determining actual self-absorption rates.

For a square cuvette sample geometry, the probability of self-absorption in the critical cones is about the same as the probability outside of the critical cones. The self-absorption probability $r$ is calculated in three different ways. We will assume that the characteristic length approximation of Eq. (9) applies for the spectral overlap calculation, with the length determined by the path length through the sample from the excitation source to the detector. We will use Eq. (12a) to find $r \eta$ from the reduced anisotropy measurements. If $R A_{\max }$ is the maximum reduced anisotropy $(2 e / 5)$ measured at low concentrations and short path lengths, the

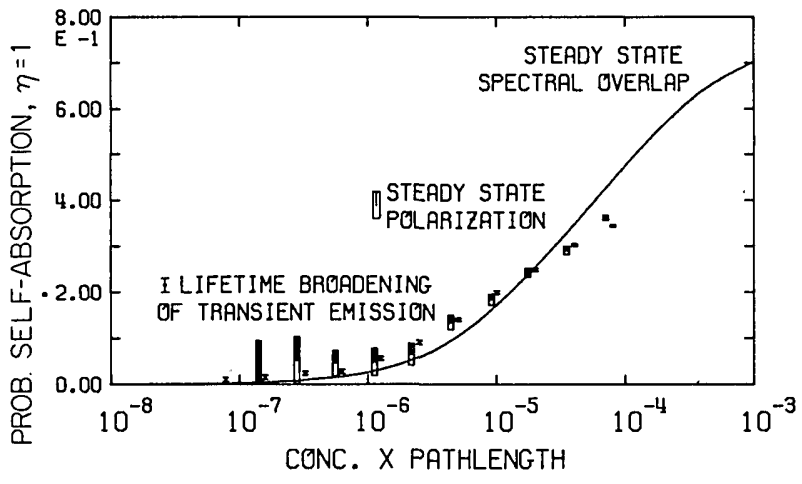

Fig. 25. Self-absorption probabilities for rhodamine-575. This shows a juxtaposition of the predicted self-absorption probabilities for the three measurement methods: spectral overlap convolution (solid curve); emission depolarization (error boxes); and transient lifetime (error bars). Lifetime measurements have the highest precision, especially at low concentrations, and the lifetime and polarization experiments are in good agreement. These two techniques actually measure the self-absorption probability times the quantum efficiency of luminescence, which we have approximated as being unity.

product of the self-absorption probability and the quantum efficiency $r \eta$ from Eq. (12a) is

$$
r \eta=\frac{1-\left(R A_{\exp } / R A_{\max }\right)}{1-\left(R A_{\exp } \cdot R A_{\max }\right)} \simeq 1-\left(R A_{\exp } / R A_{\max }\right) .
$$

Similarly, from Eq. (14), if $\tau_{\min }$ was the shortest total lifetime measured at low concentrations and short path lengths, the same result should be given by the relation $(r=\bar{r}), \quad r \eta=1-\tau_{\min } / \tau_{\exp }, \quad$ where $\tau_{\exp }$ is the measured lifetime. If we apply the preceding two relations to the measured transient emission and steady-state depolarization measurements on rhodamine-575, we obtain the results shown in Fig. 25. We have plotted the calculated product of the quantum efficiency and self-absorption probability as a function of path length times the concentration of the sample through which the emission had to travel. The solid line is the result from the characteristic length approximation of Eq. (9). This is actually just the self-absorption probability and not the product of the self-absorption with the quantum efficiency. There is very good agreement between the lifetime lenghtening and the emission depolarization measurements. The characteristic length calculation overestimates the self-absorption rate at high optical densities, as we showed earlier in Fig. 23. The measured value of $R A_{\max }=0.18$ for rhodamine- 575 in glycol is consistent with a rotational diffusion time of several nanoseconds, which is the rate observed for similar dyes in glycol. ${ }^{11}$

\section{Optimal Efficiency}

We know all the characteristics of rhodamine- 575 which determine the efficiency of a single-dye LSC, so that now it is instructive to determine the optimal efficiency of an LSC containing rhodamine-575 as a function of its size. Since the optimal concentration of 


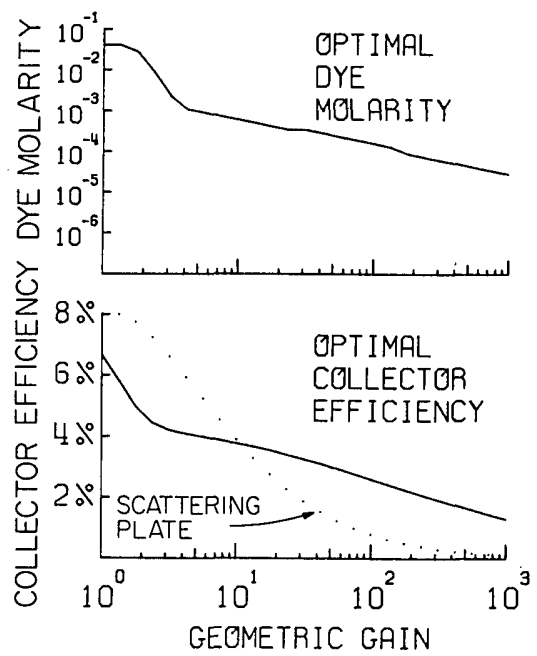

Fig. 26. Optimal efficiency and dye concentration for a single-dye LSC. Geometry assumed is an infinite ribbon with $18 \%$ AM1 silicon cells mounted on both edges. Plate has an index of 1.49 and contains rhodamine- 575 with an assumed quantum efficiency of luminescence of 0.9 . Dotted line represents the performance of a scattering plate with an identical geometry.

the dye will vary with size, we also must compute the concentration. We use the PSC geometry formalism from LSC-1 [Eqs. (11) and (20)], to compute the first generation collection efficiency $Q(1)$. We used the characteristic length approximation of Eq. (9) to compute the self-absorption rate in the critical cones. These were combined in Eq. (1) to give the total collection efficiency with the approximation of no reflection losses and only homogeneous spectral broadening. We will assume edge-mounted cells which have $18 \%$ AM1 efficiency coupled to a plate with an index of 1.49. The quantum efficiency of the dye is assumed to be 0.9 . The result of this calculation is given in Fig. 26. For the sake of comparison, the system efficiency of a scattering plate with an identical geometry is included. The single-dye plate outperforms the scattering plate for geometric gains greater than 10. From Table I, we find that the CODE of rhodamine-575 is about 20. Thus, for a plate with a gain of 20 , we expect a collection efficiency of $\sim 0.5$ and that $\sim 40 \%$ of the solar flux to which the cell would be sensitive would be absorbed by the plate. The zeroth-order estimate for the efficiency of the plate for a gain of 20 is therefore $0.18 \times 0.5 \times 0.4=$ $0.036(3.6 \%)$ in agreement with Fig. 26.

Finally, the simplest model for the efficiency of an LSC which includes self-absorption effects can be made as an extension of a simplified solar cell efficiency model. We assume that all light with energy higher than some absorption cutoff $E_{i}$ is absorbed and reemitted with an energy $E_{c}$ minus the Stokes shift. This is transmitted to the cells, which then subtract another $0.4 \mathrm{eV}$ before producing useful electricity. If we assume a $0.7 \mathrm{eV}$ Stokes shift (typical for DCM), this model predicts $9 \%$ maximum overall efficiency using either silicon or gallium arsenide cells. ${ }^{12}$

\section{Conclusions and Ramifications}

We can draw five principal conclusions from these studies:

(1) It is important to know the relative homogeneity of the spectral broadening of the absorption and emission lines for the luminescing species in an LSC. We have found that our organic laser dye rhodamine- 575 is markedly inhomogeneously broadened when dissolved in methyl methacrylate monomer and polymerized, while dye solutions and dye diffused into precast material are predominantly homogeneously broadened. The preferable case is the one that produces the least low energy absorption tail. Materials for altering both homogeneous and inhomogeneous linewidths are presently under investigation.

(2) We have demonstrated three different techniques for measuring the effect of self-absorption in sample platios. These techniques are spatial filtering, emission depolarization, and lifetime lengthening. There is good agreement between the techniques when measuring the self-absorption rates of rhodamine-575. Each technique is best suited for different applications. The lifetime lengthening measurement is the most accurate, especially at the low concentrations and path lengths. The spectral overlap is best suited for calculating the performance of a plate. The emission depolarization measurement requires a minimal expenditure for equipment and is quite accurate for high rates of selfabsorption.

(3) Self-absorption plays a fundamental role in determining LSC efficiencies in large plates or plates with a high geometric gain. We defined the CODE to be the peak optical density of a sample so that emission passing through the sample has a $50 \%$ chance of being selfabsorbed in the sample. Tabulated critical optical densities for eighteen principal laser dyes are shown in Table I. We have shown that the CODE is roughly the maximum geometric gain at which the collection efficiency of the plate is greater than or equal to 0.5 for typical dyes and matrix materials. None of the dyes tested could successfully perform as single-dye LSCs or as final dyes in a multiple-dye LSC with a system efficiency of $>8 \%$ assuming a geometric gain of greater than 100 and that the edge-mounted solar cells have an 18\% AM1 efficiency.

(4) We suggest that the CODE allow for a rough calculation of the efficiency of an LSC plate, including self-absorption effects. Thus the CODE of a dye is an important parameter in selecting a dye for use in an LSC. As an example of such a calculation, let us consider a plate of thickness $T$ having a geometric gain (equal to the area of the face divided by the active area of the edge) and containing a dye with a quantum efficiency of luminescence $\eta$, a concentration $C$, a normalized luminescence spectrum $f(\bar{\nu})$, and an extinction coefficient $\epsilon(\bar{\nu})$ with a peak at $\bar{\nu}_{\max }$. We will assume that the concentration of the dye is adjusted so that the peak optical density across the thickness of the plate is one. The CODE of this dye is defined to be CODE = $\epsilon\left(\bar{\nu}_{m}\right) C X$, where $X$ is an arbitrary path length chosen so that

$$
1 / 2=\int_{0}^{\infty} f(\bar{\nu}) d \bar{\nu} 10^{-\epsilon(\bar{\nu}) C X} .
$$


We have shown that for a plate whose geometric gain is equal to this CODE and which has an index of refraction of $\sim 1.5, \sim 50 \%$ of the emission can be collected by edge-mounted absorbers. The collection efficiency therefore is approximately $Q=\eta / 2$. The fraction of the sunlight absorbed is $S / I[$ LSC-1, Eq. (11)]:

$$
S / I=\int_{\bar{\nu}_{a}}^{\infty} I(\bar{\nu}) d \bar{\nu}\left[1-10^{-C_{\epsilon}(\bar{\nu}) \mathrm{T}}\right] / \int_{\bar{\nu}_{a}}^{\infty} I(\bar{\nu}) d \bar{\nu},
$$

where $I(\bar{\nu})$ is the solar flux and $\bar{\nu}_{a}$ is the wave number corresponding to the low energy absorption limit for the solar cell used. $S / I$ can vary from $<10 \%$ in single-dye plates to $75 \%$ in multiple-dye plates. The total collector efficiency is

$$
\text { Eff. }=1 / 2 \eta \eta_{\mathrm{AM} 1} S / I \equiv(S / I) \mathrm{Q} \eta_{\text {cell }},
$$

where $\eta_{\text {AM1 }}$ is the air mass 1 efficiency of the solar cells used.

For example, from Table I we find that rhodamine590 has a CODE of 25 , a quantum efficiency of 0.95 , and a peak extinction coefficient of $107,000 \mathrm{moles} / \mathrm{cm} / \mathrm{liter}$. If we make a $3-\mathrm{mm}$ thick Plexiglas plate with a geometric gain of 25 containing $30-\mu \mathrm{M}$ rhodamine-590, if the solar cells attached to the edge of the plate have an $18 \%$ AM1 efficiency, and if we assume that the dye initially absorbs $20 \%$ of the usable sunlight, the above relation lets us approximate the total collector efficiency as $0.98 \times 0.18 \times 0.2 / 2=1.8 \%$, which is typical for a plate of this type.

(5) Dye degradation is now the most critical technical barrier to the practical utilization of the LSC. The degradation rates appear more dependent on the total flux than on UV intensity alone. Preliminary studies performed here show results that are consistent with previously published quantum efficiencies for photodegradation, which usually are within an order of magnitude of $10^{-6}$. We are optimistic about the possibility of increasing the lifetime of the dyes, since in some cases, lifetimes of at least a year are measured.

A. H. Zewail is an Alfred P. Sloan Fellow and Camille and Henry Dreyfus Teacher-Scholar. Part of this work was supported by a contract from the U.S. Department of Energy and part by ARCO Solar, Inc. We thank R. J. Robbins and D. P. Millar for performing the transient lifetime measurements and for their stimulating discussions. We particularly thank R. J. Robbins for providing us with the anistropies for higher-order generations. We thank Stuart Vincent for help in casting plates and in the degradation experiments and Prakash Kasiraj for writing data handling and plotting software. We also thank Jim Liu, Bob Mueller, and co-workers at JPL for performing prototype measurements. J. S. Batchelder and A. H. Zewail also hold appointments in the Department of Applied Physics.

This is Contribution 6455 from the Arthur Amos Noyes Laboratory of Chemical Physics, California Institute of Technology, Pasadena 91125.

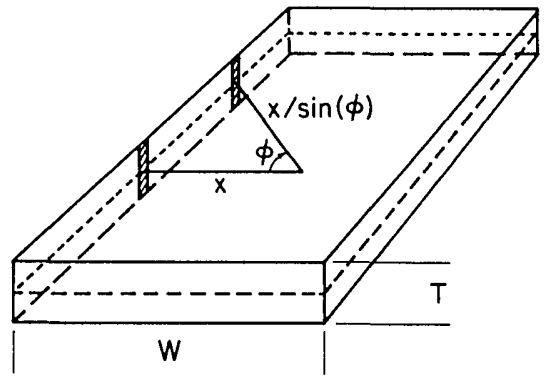

Fig. 27. Schematic diagram of a scattering plate.

\section{Appendix A: Scattering Plate}

We wish to compute the collection efficiency of a scattering plate. The geometry of interest is an infinite ribbon of thickness $T$ and width $W$, as shown in Fig. 27 . We shall assume that there is a plane of scattering centers located midway between the two faces of the ribbon.

The fraction of an isotropically scattered light flux incident on the scattering plane a distance $x$ away from one edge that is intercepted by that edge is

$$
\begin{aligned}
F(x) & =\frac{1}{4 \pi} \int_{-\pi / 2}^{\pi / 2} d \phi \int_{a_{-}}^{a_{+}} \sin \theta d \theta \\
& =\frac{T}{2 \pi x} \int_{-\pi / 2}^{\pi / 2} d \phi \frac{\cos \phi}{\sqrt{ } 1+\left(\frac{T \cos \phi}{x}\right)^{2}}=\frac{1}{\pi} \tan ^{-1}\left(\frac{T}{x}\right),
\end{aligned}
$$

where $a_{ \pm}=\pi / 2 \pm \tan ^{-1}(T \cos \phi / x)$. We have allowed only light trajectories which do not intercept the scattering plane.

If the scattering plate has solar cells mounted on both edges, the fraction of the scattered incident light which is collected without intercepting the scattering plane a second time is

$$
\begin{aligned}
Q_{s}^{(1)} & =\frac{2}{W} \eta_{\mathrm{scat}} \int_{0}^{W} d x F(x) \\
& =\frac{2}{W \pi} \eta_{\mathrm{scat}} \int_{0}^{W} d x \tan ^{-1}\left(\frac{T}{x}\right) \\
& =\frac{2 T}{\pi W} \eta_{\text {scat }}\left\{\frac{W}{T} \tan ^{-1}\left(\frac{T}{W}\right)+1 / 2 \ln \left[1+\left(\frac{W}{T}\right)^{2}\right]\right\},
\end{aligned}
$$

where $\eta_{\text {scat }}$ is the scattering effiency of the scattering plane.

We note that if the scattered light does not escape out of the critical cones, and if it is not collected by the solar cells, it must intercept the scattering plane again. Assuming that the spatial distribution of this scattered light is isotropic, we find that the higher generation collection efficiencies are

$$
\begin{aligned}
& Q_{s}^{(2)}=(1-P)\left[\eta_{\text {scat }}-Q_{s}^{(1)}\right] Q_{s}^{(1)}, \\
& Q_{s}^{(3)}=(1-P)^{2}\left[\eta_{\text {scat }}-Q_{s}^{(1)}\right]^{2} Q_{s}^{(1)} .
\end{aligned}
$$

Therefore the total probability of the solar cells collecting the scattering incident light is

$$
Q_{s}=\sum_{i} Q_{s}^{(i)}=\frac{Q_{s}^{(1)}}{1-(1-P)\left[\eta_{s}-Q_{s}^{(1)}\right]} .
$$


The dotted line in Fig. 26 shows the performance of a scattering plate as a function of size. The scattering plane is in a 1-cm thick ribbon of infinite length and variable width; $18 \%$ AM1 cells are attached to both edges. The scattering efficiency is assumed to be $90 \%$.

\section{Appendix B. Higher Generation Anisotropies}

We wish to derive the reduced polarization anisotropy or the fraction of the detected emission intensity which is purely polarized in the direction of the polarized initial excitation for all generations of emission. This can be computed for low rates of self-absorption with the approximation that the absorption and emission dipoles are parallel.

Following the notation of Gordon, ${ }^{4}$ let $\hat{\epsilon}$ be a unit vector in the direction of the incident electric field polarization, $\hat{\mu}_{a i}$ a unit vector parallel to the absorption dipole of an $i$ th-generation excitation, $\hat{\mu}_{e i}$ a unit vector parallel to the corresponding emission dipole moment, and $\hat{\epsilon}_{i}$ the emission polarization direction. The angular dependence of absorption is given by the square of the projection of the incident polarization on the dipole averaged over all orientations of both $\left[\hat{\epsilon}_{i-1} \cdot \hat{\mu}_{a i}\right]^{2}$. In the radiation field limit (average distance between interacting dipoles $\gg 5000 \AA$ ), the angular distribution of the emission is also proportional to the square of the projection of the emitting dipole on a random vector $\hat{\epsilon}_{i}$ $\left[\hat{\mu}_{e i} \cdot \hat{\epsilon}_{i}\right]^{2}$. Thus the second-generation parallel and perpendicular emission intensities are

$$
\begin{aligned}
\left.I\right|^{(2)} & =K \cdot\left\langle\left[\hat{\epsilon} \cdot \hat{\mu}_{a 1}\right]^{2}\left[\hat{\mu}_{e 1} \cdot \hat{\epsilon}_{1}\right]^{2}\left[\hat{\epsilon}_{1} \cdot \hat{\mu}_{a 2}\right]^{2}\left[\hat{\mu}_{e 2} \cdot \hat{\epsilon}\right]^{2}\right\rangle, \\
I_{\perp}^{(2)} & =\frac{K}{2} \cdot\left\langle\left[\hat{\epsilon} \cdot \hat{\mu}_{a 1}\right]^{2}\left[\hat{\mu}_{e 1} \cdot \hat{\epsilon}_{1}\right]^{2}\left[\hat{\epsilon}_{1} \cdot \hat{\mu}_{a 2}\right]\left[\hat{\mu}_{e 2} \times \hat{\epsilon}\right]^{2}\right\rangle .
\end{aligned}
$$

We use the identity that the cosine of the angle $\psi$ between two vectors with orientations $\left(\theta_{1}, \phi_{1}\right)$ and $\left(\theta_{2}, \phi_{2}\right)$ is $\cos \psi=\cos \theta_{1} \cos \theta_{2}+\sin \theta_{1} \sin \theta_{2} \cos \left(\phi_{1}-\phi_{2}\right)$. In the far-field limit, the parallel and perpendicular intensities become

$$
\begin{aligned}
I I^{(2)}= & \frac{K}{(4 \pi)^{3}} \int_{0}^{2 \pi} d \phi \int_{0}^{\pi} d \theta \sin \theta \int_{0}^{2 \pi} d \phi_{1} \\
& \times \int_{0}^{\pi} d \theta_{1} \sin \theta_{1} \int_{0}^{2 \pi} d \phi_{2} \int_{0}^{\pi} d \phi_{2} \sin \theta_{2} \\
& \times \cos ^{2} \theta \cdot\left[\cos \theta \cos \theta_{1}+\sin \theta \sin \theta_{1} \cos \left(\phi-\phi_{1}\right)\right]^{2} \\
& \times\left[\cos \theta_{1} \cos \theta_{2}+\sin \theta_{1} \sin \theta_{2} \cos \left(\phi_{1}-\phi_{2}\right)\right]^{2} \cos ^{2} \theta_{2} \\
= & K \cdot 47 / 15^{3} \\
I_{\perp}^{(2)}= & \frac{K}{2(4 \pi)^{3}} \int_{0}^{2 \pi} d \phi \int_{0}^{\pi} d \theta \sin \theta \int_{0}^{2 \pi} d \phi_{1} \\
& \times \int_{0}^{\pi} d \theta_{1} \sin \theta_{1} \int_{0}^{2 \pi} d \phi_{2} \int_{0}^{\pi} d \theta_{2} \sin \theta_{2} \\
& \times \cos { }^{2} \theta\left[\cos \theta \cos \theta_{1}+\sin \theta \sin \theta_{1} \cos \left(\phi-\phi_{1}\right)\right]^{2} \\
& \times\left[\cos \theta_{1} \cos \theta_{2}+\sin \theta_{1} \sin \theta_{2} \cos \left(\phi_{1}-\phi_{2}\right)\right]^{2}\left(1-\cos ^{2} \theta_{2}\right) \\
= & K \cdot 39 / 15^{3} .
\end{aligned}
$$

We have assumed that the absorption and emission dipoles are both parallel and fixed in space on a time scale of the excitation lifetime. Thus the second generation reduced isotropy is

$$
r^{(2)}=\frac{I \|^{(2)}-I_{\perp}^{(2)}}{I \|^{(2)}+2 I_{\perp}^{(2)}}=\left(\frac{2}{5}\right)^{3} .
$$

Similarly the $i$ th generation reduced anisotropy is

$$
r^{(i)}=\left(\frac{2}{5}\right)^{(2 i-1)} \text {. }
$$

The radiation field limit is applicable for dye concentrations used in LSCs. The 50\% self-absorption probability occurs for concentration times path length of typically $10^{-4} \mathrm{~mole} \mathrm{~cm} / \mathrm{liter}$, so that the average distance for self-absorption is of the order of a centimeter for typical concentrations.

The first-generation reduced anisotropy is $r^{(1)}=2 / 5$ $=0.4$, the second is $r^{(2)}=(2 / 5)^{3}=0.064$, and the third is $r^{(3)}=(2 / 5)^{5} \cong 0.01$. Thus it is reasonable to ignore the polarization contribution from higher-order generations under these conditions.

\section{Appendix C: Self-Absorption and Collection Efficiency of an LSC Rod: Derivation of Eqs. (6) and (8)}

In LSC-1 we developed analytical expressions for the intensity and spectrum of light emerging from a blackened LSC rod including self-absorption effects [Eqs. (32)-(38)]. We wish to extend this treatment to the case where light incident on the surface of the LSC material at an angle greater than the critical angle $\theta_{c}$ is totally internally reflected.

Consider a polished semi-infinite rod of LSC material with a diameter $d$. The probability $P$ that isotropic emission in the rod will escape is

$$
P=\int_{\pi / 2-\theta_{c}}^{\pi / 2+\theta_{c}} \sin \theta d \theta / \int_{0}^{\pi} \sin \theta d \theta=\sin \theta_{c}=1 / n,
$$

where $n$ is the index of refraction.

Suppose the rod contains a luminescing dye at a concentration of $c$ moles/liter, with an extinction coefficient $\epsilon(\bar{\nu})$, with a normalized luminescence spectrum $f(\bar{\nu})$, and with a quantum efficiency of luminescence $\eta$. Furthermore, suppose that the matrix material has a small and wave number independent absorption coefficient $S C$ (or scattering coefficient for light which scatters out of the rod). In general the transmission through a length $(a)$ of this material is given by the Beers-Lambert law:

$$
T(a, \bar{\nu})=\exp \{-a[\ln (10) \cdot C \cdot \epsilon(\bar{\nu})+S C]\} .
$$

If the scattering coefficient is small, it can be ignored over short path lengths. Then the transmission through an incrementally longer distance is

$$
\begin{aligned}
T(a+\Delta, \bar{\nu}) & =T(a, \bar{\nu}) \exp [-\Delta \cdot \ln (10) \cdot C \cdot \epsilon(\bar{\nu})] \\
& =T(a, \bar{\nu})[1-\Delta \cdot \ln (10) \cdot C \cdot \epsilon(\bar{\nu})] .
\end{aligned}
$$

The probability of absorption in the incremental distance is the difference in the two transmission probabilities:

$$
\begin{aligned}
T(a, \bar{\nu})-T(a+\Delta, \bar{\nu})= & \Delta \cdot \ln (10) \cdot C \cdot \epsilon(\bar{\nu}) \\
& \cdot \exp \{-a[\ln (10) \cdot C \cdot \epsilon(\bar{\nu})+S C\} .
\end{aligned}
$$

Now we go from a 1- to a 3-D case, which requires that we average over all possible paths. Suppose a molecule 
located on the symmetry axis of the rod emits a photon whose path makes an angle $\theta$ with the symmetry axis. We will assume that all emissions from a disk element occur at the center of the disk. This is a reasonable approximation for emission path lengths which are longer than the diameter of the rod. If the emission occurs at point $y$ from the end of the rod, and we are interested in the absorption probability per unit length at $x$ from the end of the rod, the length of a trajectory will be $a / \cos \theta$, where $a=|x-y|$. Similarly the incremental distance is increased by a factor of $1 / \cos \theta$. The probability of absorption per unit length becomes

$$
\begin{aligned}
\Lambda(x, y, \bar{\nu})= & \int_{0}^{B^{\prime}} \frac{T\left(\frac{a}{\cos \theta}, \bar{\nu}\right)-T\left(\frac{a+\Delta}{\cos \theta}, \bar{\nu}\right)}{\Delta} \sin \theta d \theta \\
= & \int_{0}^{B^{\prime}} d \theta \frac{\sin \theta}{\cos \theta} \ln (10) \cdot C \cdot \epsilon(\bar{\nu}) \\
& \times \exp \{-|x-y|[\ln (10) \cdot C \cdot \epsilon(\bar{\nu})+S C\}, \\
B^{\prime}= & \begin{cases}\pi / 2-\theta_{c} & \text { for }|x-y|>\frac{d}{2} \tan \theta_{c}, \\
\tan ^{-1}\left(\frac{d}{2|x-y|}\right) \text { for }|x-y|<\frac{d}{2} \tan \theta_{c} .\end{cases}
\end{aligned}
$$

The upper limit of integration depends on the distance $|x-y|$. If, for example, $|x-y|<(d / 2) \tan \theta_{c}$, light from $y$ with a polar angle greater than $\pi / 2-\theta_{c}$ can reach $x$, even though it would not be trapped by total internal reflection. Now let

$$
\begin{aligned}
z & =|x-y| \cdot[\ln (10) \cdot C \cdot \epsilon(\bar{\nu})+S C] / \cos \theta, \\
\frac{d z}{z} & =\frac{\sin \theta}{\cos \theta} d \theta .
\end{aligned}
$$

Substituting in Eq. (C2) gives

$$
\begin{aligned}
\Lambda(x, y, \bar{\nu})= & \int_{A}^{B} d z \frac{\exp (-z)}{z} \ln (10) \cdot C \cdot \epsilon(\bar{\nu}), \\
A= & |x-y| \cdot[\ln (10) \cdot C \cdot \epsilon(\bar{\nu})+S C], \\
B= & \left\{\begin{array}{l}
|x-y| \cdot[\ln (10) \cdot C \cdot \epsilon(\bar{\nu})+S C] / \sin \theta_{c} \text { when }|x-y|>\frac{d}{2} \tan \theta_{c}, \\
{[\ln (10) \cdot C \cdot \epsilon(\bar{\nu})+S C] \sqrt{\frac{d^{2}}{4}+(x-y)^{2}} \text { when }|x-y|<\frac{d}{2} \tan \theta_{c} .}
\end{array}\right.
\end{aligned}
$$

Finally, we perform a weighted average over the luminescence spectrum to arrive at Eq. (6):

$$
\Lambda(x, y)=\int_{0}^{\infty} d \bar{\nu} f(\bar{\nu}) \Lambda(x, y, \bar{\nu}) .
$$

To derive Eq. (8), we first observe that the emission at $x$ and $\bar{\nu}$ along the rod is the sum of all generations of excitations at $x$ times the normalized luminescence spectrum times the quantum efficiency

$$
\eta f(\bar{\nu}) \sum_{i=1}^{\infty} \Xi^{(i)}(x) .
$$

Emission off-axis by an angle of $\theta$ is attenuated by

$$
T\left(\frac{x}{\cos \theta}, \bar{\nu}\right)=\exp \{-x[\ln (10) \cdot C \cdot \epsilon(\bar{\nu})+S C] / \cos \theta\} .
$$

The output spectrum is the average of this emission over all elements $x$ and all trajectories of trapped emission $0 \leq \theta \leq \pi / 2-\theta_{c}$ :

$$
\begin{aligned}
Q(\bar{\nu})= & \int_{0}^{\pi / 2-\theta_{c}} d \theta \sin \theta \int_{0}^{\infty} d x \\
& \cdot \exp \{-x[\ln (10) \cdot C \cdot \epsilon(\bar{\nu})+S C] / \cos \theta\} \\
& \cdot \eta \cdot f(\bar{\nu}) \cdot \int_{i=1}^{\infty} \Xi^{(i)}(x) .
\end{aligned}
$$

This is Eq. (8). Summing over wave numbers gives the overall collection efficiency:

$$
Q=\int_{0}^{\infty} d \bar{\nu} Q(\bar{\nu})
$$

Note added in proof. Recent experiments on some dyes in PMMA by A. Gupta and S. Vincent indicate that the value of $\sim 0.2$ for the reduced anisotropy of emission in our samples is due to the presence of unpolymerized monomers in the vicinity of the dye. This allows rotational diffusion (D. Millar, R. Shah and A. Zewail, Chem. Phys. Letts. 66, 435 (1979)) during the fluorescence lifetime. The implications of these important findings to photostability is currently under investigation.

\section{References}

1. J. S. Batchelder, A. H. Zewail, and T. Cole, Appl. Opt. 18, 3090 (1979) and references therein.

2. Exciton Chemical Co., Inc., P.O. Box 3204, Overlook Station, Dayton, Ohio 45431.

3. Acrilex, Inc., 8 Hope Street, Jersey City, N.J. 07307.

4. R. G. Gordon, J. Chem. Phys. 45, 1643 (1966).

5. R. J. Robbins, D. M. Millar, and A. H. Zewail, J. Chem. Phys. 75, 3649 (1981). [A brief description of the apparatus appears in $R$. J. Robbins, D. P. Millar, and A. H. Zewail, Picosecond Phenomena II, Springer Topics in Chemical Physics 14, R. M. Hochstrasser, W. Kaiser, and C. V. Shank, Eds. (Springer, Berlin, 1980).]

6. D. Beer and J. Weber, Opt. Commun. 5, 307 (1972).

7. R. E. Sah, G. Baur, and H. Keller, Appl. Phys. 23, 369 (1980).

8. For a planar LSC a characteristic path length can be defined that reduces the 3-D problem to a pseudo-1-D problem.

9. J. N. Demas and G. A: Crosby, J. Phys. Chem. 75, 991 (1971).

10. T. Tao, Biopolymers 8, 609 (1969).

11. A. Von Jena and H. E. Lessing, Chem. Phys. 40, 245 (1979).

12. J. S. Batchelder, Ph.D. thesis, Calif. Inst. Tech. (1981). 